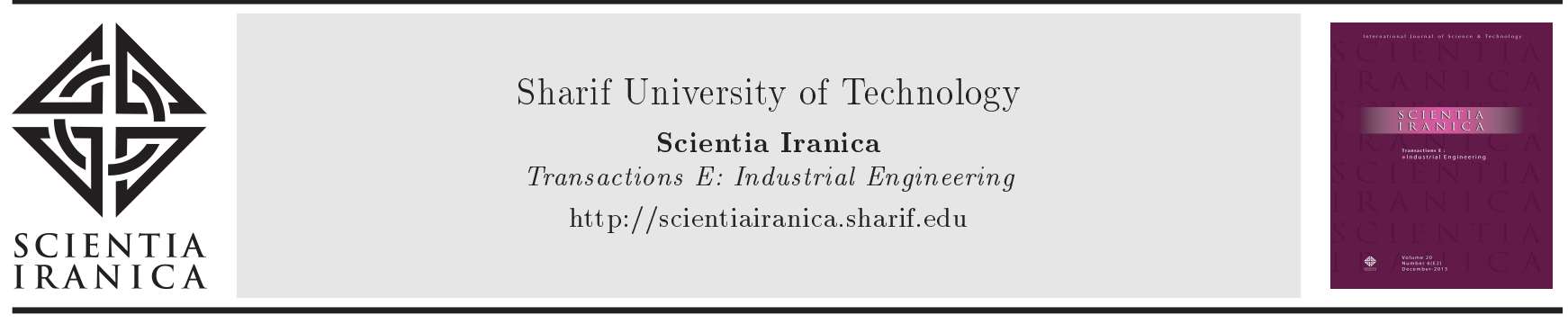

\title{
Efficiency evaluation of a three-stage leader-follower model by data envelopment analysis with double-frontier viewpoint
}

\author{
E. Vaezi ${ }^{a}$, S.E. Najafia,*, S.M. Hajimolana ${ }^{a}$, F. Hosseinzadeh Lotfi ${ }^{b}$, and \\ M. Ahadzadeh Namin ${ }^{c}$ \\ a. Department of Industrial Engineering, Science and Research Branch, Islamic Azad University, Tehran, Iran. \\ b. Department of Mathematics, Science and Research Branch, Islamic Azad University, Tehran, Iran. \\ c. Department of Mathematics, Shahr.e Qods Branch, Islamic Azad University, Tehran, Iran.
}

Received 8 October 2018; received in revised form 4 May 2019; accepted 20 May 2019

\section{KEYWORDS}

Data envelopment analysis;

Three-stage processes;

Game theory;

Goal programming;

Double-frontier;

Undesirable output.

\begin{abstract}
In this paper, a three-stage network with optimal desirable and undesirable inputs and outputs has been taken into consideration. This network is comprised of a leader and two followers. Four diverse models of Data Envelopment Analysis (DEA) to measure the efficiency or performance of this three-stage network have been taken under consideration. These are, namely, a Black Box Model and three Stackelberg Game (theory) Models. A multiplicative DEA, with a double-frontier approach, to measure the efficiency of the entire system and the performance of the Decision Making Units (DMUs), from both optimistic and pessimistic views has been utilized. In this paper, attempts have been made to present the goals of the managers in the models. Hence, aspects of goal programming have been manipulated so as to define cooperation between the leader and followers, such that the objectives of the managers are included in the models. In actual fact, a noncooperative collaboration is deliberated upon. In addition to which, in the second and third scenarios, the leader-follower, nonlinear models are present. Thereby, a heuristic approach is suggested to convert the nonlinear models into linear ones.
\end{abstract}

(C) 2021 Sharif University of Technology. All rights reserved.

\section{Introduction}

Data Envelopment Analysis (DEA) is a non-parametric method to measure the relative efficiency of a set of analogous Decision-Making Units (DMUs), with multiple inputs and outputs [1]. This method considers a frontier or boundary function, which surrounds and

\footnotetext{
*. Corresponding author.

E-mail addresses: ehsan.vaezi@srbiau.ac.ir, (E. Vaezi); e.najafi@srbiau.ac.ir (S.E.Najafi); Molana@srbiau.ac.ir (S.M. Hajimolana); farhad@hosseinzadeh.ir (F.

Hosseinzadeh Lotfi); mahnazahadzadehnamin@gmail.com (M. Ahadzadeh Namin)
}

doi: $10.24200 /$ sci.2019.51980.2459 involves factors, such as inputs and outputs. It not only determines the most efficient units, but also analyzes the inefficient ones [2]. Charnes et al. [3] developed the initial DEA task of Farrell [4]. The said model was known as (Charnes-Cooper-Rhodes) or the "CCR model". Banker et al. [5] expanded on the DEA models and presented the (Banker-Charnes-Cooper) or the "BCC model". Classical DEA models, such as CCR and BCC, assume that the systems are considered as black boxes; and due to the shortcomings in considering the intermediary variables and the internal interactions of the system, valuable information is eliminated [6]. Färe et al. [7] specified the disadvantages and weak points of the classical DEA models and referred to the Network Data Envelopment Analysis (NDEA) model. 
NDEA systems can simulate the internal structures of systems by taking the stages and sub-divisions into consideration, thereby, elevating the efficiency measurement $[8,9]$. After the introduction of NDEA models, ample studies were carried out and rendered on the grounds of various models. Over the past few years, new discussions have been presented in regard to network analysis, in view of game theory, such that this theory has become one of the vital methods in analyzing NDEA, or have been converted into multistage models [10]. Li et al. [11] presented a model for a two-stage structure, a phase which holds a more important standpoint for managers; they named this phase "leader" and the other "follower". In order to calculate efficiency, initially, the efficiency of the leader phase was maximized to the optimum and thus, the efficiency of the follower phase was secured by maintaining a constant efficiency in the phase of the leader. This exemplary was designated as a decentralized control or a Stackelberg Game, which has been widely utilized by researchers in recent years. An et al. [12] procured a network which is comprised of two stages, having a collaborative condition between them, and computed the efficiency of this network, under cooperative and non-cooperative conditions on a (leader-follower) basis. Results demonstrated that the overall efficiency in cooperative conditions was higher than that of the non-cooperative ones. In another similar research, Wu et al. [13] contemplated and computed the efficiency of a two-stage network, under undesirable outputs in cooperative and non-cooperative conditions. The results of this research, which considers total efficiency as the sum of the efficiency component, denotes that the efficiency of the sub-DMUs is in the condition of a leader in the maximal and as a follower in the minimal. In yet another research by Zhou et al. [14], a network consisting of a leader and some followers were evaluated in a black box and non-cooperative modes and the results were compared. In this study which aimed at minimizing costs, the CCR data envelopment analysis model was utilized. A research performed by $\mathrm{Du}$ et al. [15] on the grounds of leader-follower analyzed a parallel network in the cooperative and noncooperative modes. In recent years, the Stackelberg Game was utilized by several researchers such as Fard and Hajaghaei-Keshteli [16,17], Fathollahi-Fard et al. $[18,19]$, and Hajaghaei-Keshteli and FathollahiFard [20].

Undesirable factors are a critical area that account for DEA. For the first time, Färe et al. [21] took the undesirable factors under consideration to evaluate the efficiency in DEA models. Lu and Lo [22] classified the undesirable outputs within a framework of three modes; the first method was to overlook all the undesirable outputs. The second method was to restrict the expansion of the undesirable outputs, or these undesirable outputs were to be considered as a nonlinear DEA model; whereas, the third technique, for the undesirable outputs, was as an input, signified with a negative sign, as an output and/or was handled by imposing a single downward conversion. In the past few years, the role of the undesirable factors in DEA models has made considerable progress and the tasks of Wang et al. [23] and Wu et al. [24] are significant in this respect.

Most research carried out in the field of DEA is in static environments; and for the first time, Sengupta [25] performed efficiency evaluations in dynamic environments. In dynamic models, each time period is considered a decision-making unit. Similarly, the correlation between the time periods in these models is contemplated by using additional inputs and outputs in between these periods [26]. Since the era of Sengupta's work, until now, many articles have been published in the sphere of dynamic networks; the difference of which lies in the case studies and the manner in which the efficiency of the DMUs are computed. These include the Kawaguchi et al. [27] and Wang et al. [28] models which can be indicated in a dynamic mode respectively, for the evaluation of performance in hospital environments and banks.

DEA with a double-frontier is researched on two frontiers to compute the efficiency for each DMU. One is the efficient frontier and the other, the inefficient one. The efficiency calculated by the efficient frontier is called optimistic efficiency, whereas the efficiency computed by the inefficient frontier is known as pessimistic efficiency [29]. In the optimistic view, each DMU is compared with a set of efficient units that are on the efficient frontier and in the pessimistic view each DMU is compared with a set of inefficient units that are on the inefficient frontier [30]. In the optimistic view the amount of efficiency which has come to hand is less than (1), under the condition where the DMU under evaluation is not on the efficient frontier and equates to (1) when the DMU under evaluation is on the efficient frontier. The efficiency value gained in the pessimistic view is more than (1), on condition where the DMU under evaluation is not on the inefficient frontier and is equivalent to (1), if the DMU under evaluation is on the inefficient frontier [31]. When calculating the optimistic efficiency, the nearer the DMU proves to be to the efficiency frontier, the more desirable, whereas, in the case of computing pessimistic efficiency, the further the distance of the DMU, the better, which has additional desirability [32]. For the first time, Doyle et al. [33] computed the efficiency of DMUs from optimistic and pessimistic viewpoints. In recent years, the double-frontier was utilized by numerous researchers such as Badiezadeh et al. [34]; Azizi et al. [35] and Wang and Lan [36].

Multiple criteria decision-making can be divided 
into two groups, consisting of multi-criterion and multi-objective decision-making. Goal Programming (GP) is one of the multi-objective decision-making techniques, which assists in encompassing several aims synchronously; and by minimizing the deviation between these objectives, the optimal solution can be determined [37]. In this method, the objective function of the key problem is somehow formulated by the auxiliary variables that are, namely, deviations from the goal condition, so that the total set of undesirable deviations of the ideals are minimized. This technique specifies the goals achieved and the ones which have not been so. In addition to which, by utilizing a GP, the amount of deviation for each of these goals comes to hand from their ideal level [38-40]. A GP was performed by Charnes and Cooper [41] in 1961. Yousefi et al. [42] suggested a hybrid GP-DEA model in a network to present an improvement in solutions and to rank units (all efficient and inefficient ones) based on the requirements of experts. In the past few years, numerous researchers have used the GP method and rendered new models. For such paradigms, one can refer to Maiti and Roy [43,44] Chen et al. [45] Trivedi and Singh [46] He et al. [47], and Roy et al. $[48,49]$. Methods in relevance to GP modes are extremely diverse and even make provisions to optimize contradictory goals. Jolai et al. [50] set up and utilized GP for three kinds of analysis: 1-Specify the essential resources to fulfill a set of goals under consideration. 2-Determine the intensity of attaining the goals. 3Determine the optimal and substantial response with due attention to the amount of resources available and the priority of objectives or goals. Table 1 reviews the studies which have applied the game theory methods in DEA. The last row of Table 1 presents characteristics of the current paper.

The main objective of this paper is to expand DEA models, utilizing GP concepts. This task is performed for non-cooperative models (leader-follower). The weakness present in the non-cooperative models is that the leader maximizes the performance on its own. Hence, there is a probability that this may cause an annihilation of the follower. The flaw or weakness of the non-cooperative models in the case where the network is a series, illustrates itself more prominently, as under such conditions, a failure of one stage leads to the collapse of the entire system. The other reason is that, in this case, the efficiency or performance is computed from the multiplicative angle which is devoid of compensation properties. In order to overpower this weakness, a level of goals is defined for the followers. In fact, in this paper and on terms with the noncooperative game, collaboration is proposed between the leader and follower. In this manner, the views of the managers are induced into DEA models. A comparison is made, in view of the proposed model, with the other two non-cooperative common models and that of the black box model, so that managers can accurately analyze and secure the level of goals and the results of the proposed model. Hence, it is for this purpose that, in this paper, the efficiency of a three-stage performance is attained on the basis of four scenarios, a black box scenario and three noncooperative scenarios. An optimistic and pessimistic approach is utilized to secure efficiency and increase accuracy. In the second and third scenarios, the noncooperative models, from the optimistic and pessimistic views, cannot be turned into linear models, because of the additional inputs and outputs in the first, second and third stages. Therefore, a heuristic technique is used to convert the nonlinear models into linear models. In accordance with the points mentioned, most research performed in the network deliberates on two stages, but the current research takes a three-stage process into consideration, which, in addition to the intermediary variables, has also additional and undesirable inputs and outputs. As a summarization, the contributions of this paper are as follows:

- A three-stage network is taken under consideration with respect to additional desirable and undesirable inputs and outputs;

- The suggested model is a hybrid DEA and GP model in a network structure;

- A kind of cooperation between the leader and followers is defined, so that the objectives of the managers are capable of being inserted into the models;

- A double frontier approach is utilized to evaluate efficiency, in order to make results more realistic;

- A heuristic technique is proposed to convert nonlinear models into linear models;

- Implementation of the suggested model on an authentic example (a real world factory with a production area, a warehouse for goods and a delivery point);

- The said factory is considered a dynamic network.

The structure of this paper has been rendered as follows: Section 2 describes the model and, in this section, after introducing the structure of the network, the modeling is performed from the black box perspective and three non-cooperative scenarios. Section 3 renders the model solution and presents the same according to the heuristic method. In Section 4 a case-history is described, where a factory in the real world has been rendered and illustrated as an example. Finally, Section 5 concludes the paper.

\section{Model description}

A set of $n$ homogeneous DMUs are considered that are denoted by $D M U_{j}(j=1, \ldots, n)$, and each $D M U_{j}$ 
Table 1. Classification of studies on Data Envelopment Analysis (DEA)-game theory method.

\begin{tabular}{|c|c|c|c|c|c|c|c|c|}
\hline Reference & $\begin{array}{c}\text { Type of } \\
\text { game }\end{array}$ & $\begin{array}{c}\text { Structure } \\
\text { of network }\end{array}$ & $\begin{array}{c}\text { Additional } \\
\text { inputs }\end{array}$ & $\begin{array}{c}\text { Undesirable } \\
\text { output }\end{array}$ & $\begin{array}{c}\text { Type of } \\
\text { modelling }\end{array}$ & $\begin{array}{l}\text { Type of } \\
\text { frontier }\end{array}$ & $\begin{array}{c}\text { DEA- } \\
\text { Gp }\end{array}$ & Dynamic \\
\hline Hwang et al. [1] & Cooperative & One-stage & - & $\sqrt{ }$ & $\begin{array}{l}\text { Linear } \\
\text { programming }\end{array}$ & $\begin{array}{l}\text { Optimistic } \\
\text { view }\end{array}$ & - & - \\
\hline $\begin{array}{l}\text { Kao and } \\
\text { Hwang [53] }\end{array}$ & Cooperative & Two-stage & - & - & $\begin{array}{l}\text { Linear } \\
\text { programming }\end{array}$ & $\begin{array}{l}\text { Optimistic } \\
\text { view }\end{array}$ & - & - \\
\hline Wang et al. [28] & Cooperative & Two-stage & - & - & $\begin{array}{l}\text { Linear } \\
\text { programming }\end{array}$ & $\begin{array}{l}\text { Optimistic } \\
\text { view }\end{array}$ & - & $\sqrt{ }$ \\
\hline Kou et al. [8] & Cooperative & Two-stage & $\sqrt{ }$ & - & $\begin{array}{l}\text { Linear } \\
\text { programming }\end{array}$ & $\begin{array}{l}\text { Optimistic } \\
\text { view }\end{array}$ & - & $\sqrt{ }$ \\
\hline Li et al. [11] & $\begin{array}{l}\text { Non- } \\
\text { cooperative }\end{array}$ & Two-stage & $\sqrt{ }$ & - & $\begin{array}{l}\text { Linear } \\
\text { programming }\end{array}$ & $\begin{array}{l}\text { Optimistic } \\
\text { view }\end{array}$ & - & - \\
\hline Liang et al. [10] & $\begin{array}{l}\text { Cooperative } \\
\text { and non- } \\
\text { cooperative }\end{array}$ & Two-stage & - & - & $\begin{array}{l}\text { Linear } \\
\text { programming }\end{array}$ & $\begin{array}{l}\text { Optimistic } \\
\text { view }\end{array}$ & - & - \\
\hline Wu et al. [24] & Cooperative & Two-stage & $\sqrt{ }$ & $\sqrt{ }$ & $\begin{array}{l}\text { Linear } \\
\text { programming }\end{array}$ & $\begin{array}{l}\text { Optimistic } \\
\text { view }\end{array}$ & - & - \\
\hline An et al. [12] & $\begin{array}{l}\text { Cooperative } \\
\text { and non- } \\
\text { cooperative }\end{array}$ & Two-stage & $\sqrt{ }$ & - & $\begin{array}{l}\text { Non-linear } \\
\text { programming }\end{array}$ & $\begin{array}{l}\text { Optimistic } \\
\text { view }\end{array}$ & - & - \\
\hline Wu et al. [13] & $\begin{array}{l}\text { Cooperative } \\
\text { and non- } \\
\text { cooperative }\end{array}$ & Two-stage & $\sqrt{ }$ & $\sqrt{ }$ & $\begin{array}{l}\text { Non-linear } \\
\text { programming }\end{array}$ & $\begin{array}{l}\text { Optimistic } \\
\text { view }\end{array}$ & - & $\sqrt{ }$ \\
\hline Zhou et al. [14] & $\begin{array}{l}\text { Non- } \\
\text { cooperative }\end{array}$ & Two-stage & - & - & $\begin{array}{l}\text { Non-linear } \\
\text { programming }\end{array}$ & $\begin{array}{l}\text { Optimistic } \\
\text { view }\end{array}$ & - & - \\
\hline Du et al. [15] & $\begin{array}{l}\text { Cooperative } \\
\text { and non- } \\
\text { cooperative }\end{array}$ & Three-stage & - & - & $\begin{array}{l}\text { Linear } \\
\text { programming }\end{array}$ & $\begin{array}{l}\text { Optimistic } \\
\text { view }\end{array}$ & - & - \\
\hline Badiezadeh et al. [34] & Cooperative & Three-stage & $\sqrt{ }$ & $\sqrt{ }$ & $\begin{array}{l}\text { Linear } \\
\text { programming }\end{array}$ & $\begin{array}{l}\text { Double } \\
\text {-frontier }\end{array}$ & - & - \\
\hline Shabanpour et al. [40] & Cooperative & One-stage & - & - & $\begin{array}{l}\text { Linear } \\
\text { programming }\end{array}$ & $\begin{array}{l}\text { Double- } \\
\text { frontier }\end{array}$ & $\sqrt{ }$ & - \\
\hline Yousefi et al. [42] & Cooperative & Three-stage & $\sqrt{ }$ & - & $\begin{array}{l}\text { Non-linear } \\
\text { programming }\end{array}$ & $\begin{array}{l}\text { Optimistic } \\
\text { view }\end{array}$ & $\sqrt{ }$ & - \\
\hline Current paper & Non-cooperative & Three-stage & $\sqrt{ }$ & $\sqrt{ }$ & $\begin{array}{l}\text { Non-linear } \\
\text { programming }\end{array}$ & $\begin{array}{l}\text { Double } \\
\text {-frontier }\end{array}$ & $\sqrt{ }$ & $\sqrt{ }$ \\
\hline
\end{tabular}

has three-stages, as shown in Figure 1, where all the stages are connected together in series. The inputs of the first stage are denoted by $x_{i_{1} j}^{1}\left(i_{1}=1, \ldots, I_{1}\right)$ and the undesirable outputs of the first stage by $Y_{r_{1} j}^{1}$ $\left(r_{1}=1, \ldots, R_{1}\right)$. The intermediate measures between the first and second stages by $Z_{d_{1} j}^{1}\left(d_{1}=1, \ldots, D_{1}\right)$ and between the second and third stages by $Z_{d_{2} j}^{2}$ $\left(d_{2}=1, \ldots, D_{2}\right)$. The additional inputs and outputs of the second stage are denoted by $x_{i_{2} j}^{2}\left(i_{2}=1, \ldots, I_{2}\right)$ and $Y_{r_{2} j}^{2}\left(r_{2}=1, \ldots, R_{2}\right)$, respectively. Finally, the additional inputs of the third stage are denoted by $x_{i_{3} j}^{3}$ $\left(i_{3}=1, \ldots, I_{3}\right)$ and the outputs of the third stage by $Y_{r_{3} j}^{3}\left(r_{3}=1, \ldots, R_{3}\right) . v_{i_{1}}^{1}, v_{i_{2}}^{2}$ and $v_{i_{3}}^{3}$ are adopted as the weights of the inputs to the first, second and third 


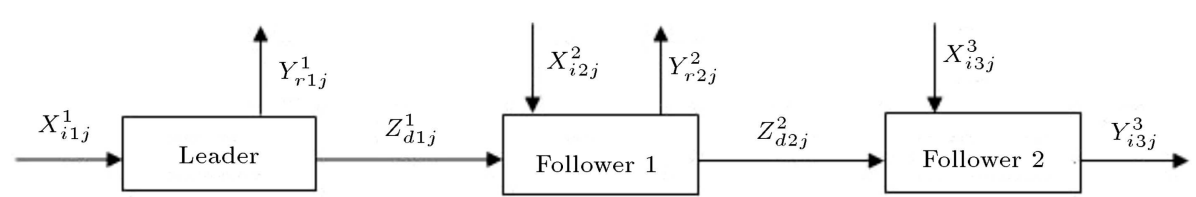

Figure 1. Structure of three-stage leader-follower system with additional inputs and undesirable outputs.

stages, respectively. $w_{d_{1}}^{1}$ and $w_{d_{2}}^{2}$ are adopted as the weights of the intermediate measures between stages 1 , 2 and 3 , respectively. The weights of the outputs for the first, second and third stages are denoted by $u_{r_{1}}^{1}$, $u_{r_{2}}^{2}$ and $u_{r_{3}}^{3}$, respectively.

In regard to analyzing the abovementioned network (Figure 1), a black box and three non-cooperative viewpoints are considered. In this section, the modeling for these four approaches is performed correspondingly. Researchers are more inclined to utilize inputoriented models for efficiency analysis, mainly for three reasons. The first is that demand reveals a growing trend, the estimation of which is an intricate matter. The second reason encompasses the fact that managers have a better control of the inputs, rather than the outputs. The third motive is that the model reflects the initial objectives of policy-makers, on the basis of being responsible for responding to the demands of the people. Furthermore, the units must reduce costs and/or restrict the use of resources. Thereby, in this research, an input-oriented model is utilized.

\subsection{Black box approach}

As indicated in the first section, the black box approach is utilized towards alleviating complex networks and ignores the intermediary variables. Figure 2 illustrates the black box model of the network and the inputs and outputs, which are the inputs and outputs of the three stages respectively.

It should be noted that, $y_{r_{1 j}}^{1}$ is an undesired output. Therefore, the efficiency of the black box approach will be described from the optimistic view, as follows:

\section{Model 1:}

$$
\theta_{o}^{\text {overall }}=\max \sum_{r_{2}=1}^{R_{2}} u_{r_{2}}^{2} y_{r_{2} o}^{2}+\sum_{r_{3}=1}^{R_{3}} u_{r_{3}}^{3} y_{r_{3} o}^{3}-\sum_{r_{1}=1}^{R_{1}} u_{r_{1}}^{1} y_{r_{1} o}^{1}
$$

s.t.

$$
\sum_{i_{1}=1}^{I_{1}} v_{i_{1}}^{1} x_{i_{1} o}^{1}+\sum_{i_{2}=1}^{I_{2}} v_{i_{2}}^{2} x_{i_{2} o}^{2}+\sum_{i_{3}=1}^{I_{3}} v_{i_{3}}^{3} x_{i_{3} o}^{3}=1
$$

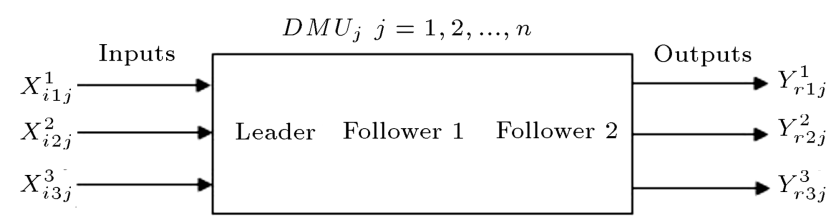

Figure 2. Structure of a "black box" system.

$$
\begin{aligned}
& \sum_{r_{2}=1}^{R_{2}} u_{r_{2}}^{2} y_{r_{2} j}^{2}+\sum_{r_{3}=1}^{R_{3}} u_{r_{3}}^{3} y_{r_{3} j}^{3}-\sum_{r_{1}=1}^{R_{1}} u_{r_{1}}^{1} y_{r_{1} j}^{1}-\sum_{i_{1}=1}^{I_{1}} v_{i_{1}}^{1} x_{i_{1} j}^{1}, \\
& \quad-\sum_{i_{2}=1}^{I_{2}} v_{i_{2}}^{2} x_{i_{2} j}^{2}-\sum_{i_{3}=1}^{I_{3}} v_{i_{3}}^{3} x_{i_{3} j}^{3} \leq 0, \quad j=1, \ldots, n, \\
& u_{r_{1}}^{1}, u_{r_{2}}^{2}, u_{r_{3}}^{3} \geq \varepsilon ; \quad r_{1}=1, \ldots, R_{1} ; \quad r_{2}=1, \ldots, R_{2} ; \\
& r_{3}=1, \ldots, R_{3}, \\
& v_{i_{1}}^{1}, v_{i_{2}}^{2}, v_{i_{3}}^{3} \geq \varepsilon ; \quad i_{1}=1, \ldots, I_{1} ; \quad i_{2}=1, \ldots, I_{2} ; \\
& i_{3}=1, \ldots, I_{3} .
\end{aligned}
$$

On the basis of Wang et al. [51] Model 1 is modified and the efficiency of the black box approach from the pessimistic view is described, as follows:

\section{Model 2:}

$$
\varphi_{o}^{\text {overall }}=\min \sum_{r_{2}=1}^{R_{2}} u_{r_{2}}^{2} y_{r_{2} o}^{2}+\sum_{r_{3}=1}^{R_{3}} u_{r_{3}}^{3} y_{r_{3} o}^{3}-\sum_{r_{1}=1}^{R_{1}} u_{r_{1}}^{1} y_{r_{1} o}^{1}
$$

s.t.:

$$
\begin{aligned}
& \sum_{i_{1}=1}^{I_{1}} v_{i_{1}}^{1} x_{i_{1} o}^{1}+\sum_{i_{2}=1}^{I_{2}} v_{i_{2}}^{2} x_{i_{2} o}^{2}+\sum_{i_{3}=1}^{I_{3}} v_{i_{3}}^{3} x_{i_{3} o}^{3}=1 \\
& \sum_{r_{2}=1}^{R_{2}} u_{r_{2}}^{2} y_{r_{2} j}^{2}+\sum_{r_{3}=1}^{R_{3}} u_{r_{3}}^{3} y_{r_{3} j}^{3}-\sum_{r_{1}=1}^{R_{1}} u_{r_{1}}^{1} y_{r_{1} j}^{1}-\sum_{i_{1}=1}^{I_{1}} v_{i_{1}}^{1} x_{i_{1} j}^{1} \\
& \quad-\sum_{i_{2}=1}^{I_{2}} v_{i_{2}}^{2} x_{i_{2} j}^{2}-\sum_{i_{3}=1}^{I_{3}} v_{i_{3}}^{3} x_{i_{3} j}^{3} \geq 0 \\
& \quad j=1, \ldots, n, \\
& u_{r_{1}}^{1}, u_{r_{2}}^{2}, u_{r_{3}}^{3} \geq \varepsilon ; \quad r_{1}=1, \ldots, R_{1} ; \quad r_{2}=1, \ldots, R_{2} ; \\
& r_{3}=1, \ldots, R_{3}, \\
& v_{i_{1}}^{1}, v_{i_{2}}^{2}, v_{i_{3}}^{3} \geq \varepsilon ; \quad i_{1}=1, \ldots, I_{1} ; \quad i_{2}=1, \ldots, I_{2} ; \\
& i_{3}=1, \ldots, I_{3} .
\end{aligned}
$$

Models 1 and 2 attain efficiency values on the basis of the distance from the efficient and inefficient frontiers, 
respectively. In respect to the Wang and Chin approach [52], the overall efficiency of Figure 2, by taking into consideration the optimistic and pessimistic views, based on the results of Models 1 and 2, described in Eq. (3) is, as follows:

$$
\emptyset_{o}=\sqrt{\theta_{o}^{\text {overall }} \cdot \varphi_{o}^{\text {overall }}}
$$

\subsection{Non-cooperative approach}

\subsubsection{First scenario}

In the first scenario, the first, second and third stages will be considered as the role of "leader", "first follower" and "second follower" respectively. The second stage is given more importance, due to its position. Hence, in this scenario, two roles are taken into consideration for the second stage:

1. Follower of the first stage;

2. Leader of the third stage.

Initially the leader stage is maximized and then, by assuming that the efficiency of the leader stage is constant or fixed, the efficiency of the second stage is maximized. Eventually, with the assumption that the two previous stages have remained constant, the efficiency of the third stage is gained. Thence, the optimistic efficiency of the leader's stage is demonstrated by $\theta_{o}^{L}$ and the optimistic efficiencies of the second and third stages by $\theta_{o}^{1 F}$ and $\theta_{o}^{2 F}$, respectively. The maximal efficiencies of the leader, second and third stages, have been defined from the optimistic standpoint, based on the approach of $\mathrm{Li}$ et al. [11], according to the following:

\section{Model 3:}

$$
\begin{aligned}
& \theta_{o}^{L *}= \\
& \max \left\{\theta_{o}^{L} \mid \theta_{j}^{L} \leq 1, \quad \theta_{j}^{1 F} \leq 1, \quad \theta_{j}^{2 F} \leq 1, \quad j=1, \ldots, n\right\} .
\end{aligned}
$$

Model 4:

$$
\begin{gathered}
\theta_{o}^{1 F *}=\max \left\{\theta_{o}^{1 F} \mid \theta_{j}^{L} \leq 1, \quad \theta_{j}^{1 F} \leq 1, \quad \theta_{j}^{2 F} \leq 1,\right. \\
\left.\theta_{o}^{L}=\theta_{o}^{L *}, j=1, \ldots, n\right\} .
\end{gathered}
$$

\section{Model 5:}

$$
\begin{gathered}
\theta_{o}^{2 F *}=\max \left\{\theta_{o}^{2 F} \mid \theta_{j}^{L} \leq 1, \quad \theta_{j}^{1 F} \leq 1, \quad \theta_{j}^{2 F} \leq 1,\right. \\
\left.\theta_{o}^{L}=\theta_{o}^{L *}, \quad \theta_{o}^{1 F}=\theta_{o}^{1 F *}, \quad j=1, \ldots, n\right\} .
\end{gathered}
$$

All the variables in Models 3, 4, and 5 are non-negative and optimum efficiency has been demonstrated with the symbol $\left(^{*}\right)$. By means of Model 3, maximum efficiency was attained by the leader stage, under the condition where the efficiencies of the other stages which came to hand are less than Model 1. In employing Model 4, maximum efficiency was gained for the second stage under circumstances in which the efficiencies of the other stages are less than Model 1 and the efficiency of the leader stage for $D M U_{o}$ is constant. In fact, under such conditions, the second stage plays the role of "follower". Finally, by utilizing Model 5, the maximum efficiency of the third stage is obtained, under such circumstances where the efficiencies of the other stages are less than Model 1 and the efficiencies of the leader and second stages remain constant for $D M U_{o}$. Under such conditions, the second stage plays the role of leader for the third stage. Models 3, 4 and 5 are fractional and by utilizing the CharnesCooper conversion (1962), as illustrated beneath, they are converted into linear models.

\section{Model 6:}

$$
\theta_{o}^{L *}=\max \sum_{d_{1}=1}^{D_{1}} w_{d_{1}}^{1} z_{d_{1} o}^{1}-\sum_{r_{1}=1}^{R_{1}} u_{r_{1}}^{1} y_{r_{1} o}^{1},
$$

s.t.:

$$
\begin{aligned}
& \sum_{i_{1}=1}^{I_{1}} v_{i_{1}}^{1} x_{i_{1} o}^{1}=1 \\
& \sum_{d_{1}=1}^{D_{1}} w_{d_{1}}^{1} z_{d_{1} j}^{1}-\sum_{r_{1}=1}^{R_{1}} u_{r_{1}}^{1} y_{r_{1} j}^{1}-\sum_{i_{1}=1}^{I_{1}} v_{i_{1}}^{1} x_{i_{1} j}^{1} \leq 0, \\
& \quad j=1, \ldots, n, \\
& \sum_{d_{2}=1}^{D_{2}} w_{d_{2}}^{2} z_{d_{2} j}^{2}+\sum_{r_{2}=1}^{R_{2}} u_{r_{2}}^{2} y_{r_{2} j}^{2}-\sum_{i_{2}=1}^{I_{2}} v_{i_{2}}^{2} x_{i_{2} j}^{2} \\
& \quad \sum_{d_{1}=1}^{D_{1}} w_{d_{1}}^{1} z_{d_{1} j}^{1} \leq 0, j=1, \ldots, n, \\
& \sum_{i_{3}}^{R_{3}} v_{i_{2}}^{3}, v_{r_{3}}^{3} y_{r_{3} j}^{3}-\sum_{i_{1}=1}^{I_{1}} v_{i_{3}}^{3} x_{i_{3} j}^{3}-\sum_{d_{2}=1}^{D_{2}} w_{d_{2}}^{2} z_{d_{2} j}^{2} \leq 0 ; i_{1}=1, \ldots, I_{1} ; i_{2}=1, \ldots, I_{2} ; \\
& r_{3}=1, \ldots, n, \\
& u_{r_{1}}^{1}, u_{r_{2}}^{2}, u_{r_{3}}^{3} \geq \varepsilon ; r_{1}=1, \ldots, R_{1} ; \quad r_{2}=1, \ldots, R_{2} ;
\end{aligned}
$$




$$
\theta_{o}^{1 F *}=\max \sum_{d_{2}=1}^{D_{2}} w_{d_{2}}^{2} z_{d_{2} O}^{2}+\sum_{r_{2}=1}^{R_{2}} u_{r_{2}}^{2} y_{r_{2} O}^{2}
$$

s.t.:

$$
\begin{aligned}
& \sum_{i_{1}=1}^{I_{1}} v_{i_{2}}^{2} x_{i_{2} o}^{2}+\sum_{d_{1}=1}^{D_{1}} w_{d_{1}}^{1} z_{d_{1} o}^{1}=1 \\
& \sum_{d_{1}=1}^{D_{1}} w_{d_{1}}^{1} z_{d_{1} j}^{1}-\sum_{r_{1}=1}^{R_{1}} u_{r_{1}}^{1} y_{r_{1} j}^{1}-\sum_{i_{1}=1}^{I_{1}} v_{i_{1}}^{1} x_{i_{1} j}^{1} \leq 0, \\
& j=1, \ldots, n, \\
& \sum_{d_{2}=1}^{D_{2}} w_{d_{2}}^{2} z_{d_{2} j}^{2}+\sum_{r_{2}=1}^{R_{2}} u_{r_{2}}^{2} y_{r_{2} j}^{2}-\sum_{i_{2}=1}^{I_{2}} v_{i_{2}}^{2} x_{i_{2} j}^{2} \\
& \quad-\sum_{d_{1}=1}^{D_{1}} w_{d_{1}}^{1} z_{d_{1} j}^{1} \leq 0, \quad j=1, \ldots, n, \\
& \sum_{r_{3}=1}^{R_{3}} u_{r_{3}}^{3} y_{r_{3} j}^{3}-\sum_{i_{1}=1}^{I_{1}} v_{i_{3}}^{3} x_{i_{3} j}^{3}-\sum_{d_{2}=1}^{D_{2}} w_{d_{2}}^{2} z_{d_{2} j}^{2} \leq 0, \\
& j=1, \ldots, n, \\
& \sum_{d_{1}=1}^{D_{1}} w_{d_{1}}^{1} z_{d_{1} o}^{1}-\sum_{r_{1}=1}^{R_{1}} u_{r_{1}}^{1} y_{r_{1} o}^{1}-\theta_{o}^{L *} \sum_{i_{1}=1}^{I_{1}} v_{i_{1}}^{1} x_{i_{1} o}^{1}=0, \\
& u_{r_{1}}^{1}, u_{r_{2}}^{2}, u_{r_{3}}^{3} \geq \varepsilon ; \quad r_{1}=1, \ldots, R_{1} ; \\
& r_{3}=1, \ldots, R_{3}, \\
& v_{i_{1}}^{1}, v_{i_{2}}^{2}, v_{i_{3}}^{3} \geq \varepsilon ; \quad i_{1}=1, \ldots, R_{2} ; \\
& i_{3}=1, \ldots, I_{3}, \\
& w_{d_{1}}^{1}, w_{d_{2}}^{2} \geq \varepsilon ; \quad d_{1}=1, \ldots, D_{1} ; \quad d_{2}=1, \ldots, D_{2} . \quad(8) \\
& i_{2}=1, \ldots, I_{2} ;
\end{aligned}
$$

\section{Model 8:}

$$
\theta_{o}^{2 F *}=\max \sum_{r_{3}=1}^{R_{3}} u_{r_{3}}^{3} y_{r_{3} o}^{3}
$$

s.t.:

$$
\begin{aligned}
& \sum_{i_{1}=1}^{I_{1}} v_{i_{3}}^{3} x_{i_{3} o}^{3}+\sum_{d_{2}=1}^{D_{2}} w_{d_{2}}^{2} z_{d_{2} o}^{2}=1 \\
& \sum_{d_{1}=1}^{D_{1}} w_{d_{1}}^{1} z_{d_{1} j}^{1}-\sum_{r_{1}=1}^{R_{1}} u_{r_{1}}^{1} y_{r_{1} j}^{1}-\sum_{i_{1}=1}^{I_{1}} v_{i_{1}}^{1} x_{i_{1} j}^{1} \leq 0 \\
& \quad j=1, \cdots, n
\end{aligned}
$$

$$
\begin{aligned}
& \sum_{d_{2}=1}^{D_{2}} w_{d_{2}}^{2} z_{d_{2} j}^{2}+\sum_{r_{2}=1}^{R_{2}} u_{r_{2}}^{2} y_{r_{2} j}^{2}-\sum_{i_{2}=1}^{I_{2}} v_{i_{2}}^{2} x_{i_{2} j}^{2} \\
& -\sum_{d_{1}=1}^{D_{1}} w_{d_{1}}^{1} z_{d_{1} j}^{1} \leq 0, \quad j=1, \cdots, n, \\
& \sum_{r_{3}=1}^{R_{3}} u_{r_{3}}^{3} y_{r_{3} j}^{3}-\sum_{i_{1}=1}^{I_{1}} v_{i_{3}}^{3} x_{i_{3} j}^{3}-\sum_{d_{2}=1}^{D_{2}} w_{d_{2}}^{2} z_{d_{2} j}^{2} \leq 0, \\
& j=1, \ldots, n, \\
& \sum_{d_{1}=1}^{D_{1}} w_{d_{1}}^{1} z_{d_{1} o}^{1}-\sum_{r_{1}=1}^{R_{1}} u_{r_{1}}^{1} y_{r_{1} o}^{1}-\theta_{o}^{L *} \sum_{i_{1}=1}^{I_{1}} v_{i_{1}}^{1} x_{i_{1} o}^{1}=0, \\
& \sum_{d_{2}=1}^{D_{2}} w_{d_{2}}^{2} z_{d_{2} o}^{2}+\sum_{r_{2}=1}^{R_{2}} u_{r_{2}}^{2} y_{r_{2} o}^{2}-\theta_{o}^{1 F *} \sum_{i_{1}=1}^{I_{1}} v_{i_{2}}^{2} x_{i_{2} o}^{2}, \\
& -\theta_{o}^{1 F *} \sum_{d_{1}=1}^{D_{1}} w_{d_{1}}^{1} z_{d_{1} o}^{1}=0, \\
& u_{r_{1}}^{1}, u_{r_{2}}^{2}, u_{r_{3}}^{3} \geq \varepsilon ; \quad r_{1}=1, \ldots, R_{1} ; \quad r_{2}=1, \ldots, R_{2} ; \\
& r_{3}=1, \ldots, R_{3} \\
& v_{i_{1}}^{1}, v_{i_{2}}^{2}, v_{i_{3}}^{3} \geq \varepsilon ; \quad i_{1}=1, \ldots, I_{1} ; \quad i_{2}=1, \ldots, I_{2} ; \\
& i_{3}=1, \ldots, I_{3} \text {; } \\
& w_{d_{1}}^{1}, w_{d_{2}}^{2} \geq \varepsilon ; \quad d_{1}=1, \ldots, D_{1} ; \quad d_{2}=1, \ldots, D_{2} .
\end{aligned}
$$

In accordance with the work of Wang et al. [51], Models 6,7 , and 8 are modified, as given below, so that the minimum efficiencies of the first, second and third stages are gained, respectively, from the pessimistic view.

\section{Model 9:}

$$
\varphi_{o}^{L *}=\min \sum_{d_{1}=1}^{D_{1}} w_{d_{1}}^{1} z_{d_{1} o}^{1}-\sum_{r_{1}=1}^{R_{1}} u_{r_{1}}^{1} y_{r_{1} o}^{1},
$$

s.t.:

$$
\begin{aligned}
& \sum_{i_{1}=1}^{I_{1}} v_{i_{1}}^{1} x_{i_{1}}^{1}=1 \\
& \sum_{d_{1}=1}^{D_{1}} w_{d_{1}}^{1} z_{d_{1} j}^{1}-\sum_{r_{1}=1}^{R_{1}} u_{r_{1}}^{1} y_{r_{1} j}^{1}-\sum_{i_{1}=1}^{I_{1}} v_{i_{1}}^{1} x_{i_{1} j}^{1} \geq 0 \\
& \quad j=1, \ldots, n,
\end{aligned}
$$




$$
\begin{aligned}
\sum_{d_{2}=1}^{D_{2}} w_{d_{2}}^{2} z_{d_{2} j}^{2} & +\sum_{r_{2}=1}^{R_{2}} u_{r_{2}}^{2} y_{r_{2} j}^{2}-\sum_{i_{2}=1}^{I_{2}} v_{i_{2}}^{2} x_{i_{2} j}^{2} \\
& -\sum_{d_{1}=1}^{D_{1}} w_{d_{1}}^{1} z_{d_{1} j}^{1} \geq 0, \quad j=1, \ldots, n,
\end{aligned}
$$$$
\sum_{r_{3}=1}^{R_{3}} u_{r_{3}}^{3} y_{r_{3} j}^{3}-\sum_{i_{1}=1}^{I_{1}} v_{i_{3}}^{3} x_{i_{3} j}^{3}-\sum_{d_{2}=1}^{D_{2}} w_{d_{2}}^{2} z_{d_{2} j}^{2} \geq 0
$$$$
j=1, \ldots, n,
$$$$
u_{r_{1}}^{1}, u_{r_{2}}^{2}, u_{r_{3}}^{3} \geq \varepsilon ; \quad r_{1}=1, \ldots, R_{1} ; \quad r_{2}=1, \ldots, R_{2}
$$$$
r_{3}=1, \ldots, R_{3} \text {, }
$$$$
v_{i_{1}}^{1}, v_{i_{2}}^{2}, v_{i_{3}}^{3} \geq \varepsilon ; \quad i_{1}=1, \ldots, I_{1} ; \quad i_{2}=1, \ldots, I_{2} ;
$$$$
i_{3}=1, \ldots, I_{3},
$$$$
w_{d_{1}}^{1}, w_{d_{2}}^{2} \geq \varepsilon ; \quad d_{1}=1, \ldots, D_{1} ; \quad d_{2}=1, \ldots, D_{2} \cdot(10)
$$

\section{Model 10:}

$$
\varphi_{o}^{1 F *}=\min \sum_{d_{2}=1}^{D_{2}} w_{d_{2}}^{2} z_{d_{2} o}^{2}+\sum_{r_{2}=1}^{R_{2}} u_{r_{2}}^{2} y_{r_{2} o}^{2}
$$

s.t.:

$$
\begin{aligned}
& \sum_{i_{1}=1}^{I_{1}} v_{i_{2}}^{2} x_{i_{2} o}^{2}+\sum_{d_{1}=1}^{D_{1}} w_{d_{1}}^{1} z_{d_{1} o}^{1}=1 \\
& \sum_{d_{1}=1}^{D_{1}} w_{d_{1}}^{1} z_{d_{1} j}^{1}-\sum_{r_{1}=1}^{R_{1}} u_{r_{1}}^{1} y_{r_{1} j}^{1}-\sum_{i_{1}=1}^{I_{1}} v_{i_{1}}^{1} x_{i_{1} j}^{1} \geq 0
\end{aligned}
$$$$
j=1, \ldots, n,
$$$$
\sum_{d_{2}=1}^{D_{2}} w_{d_{2}}^{2} z_{d_{2} j}^{2}+\sum_{r_{2}=1}^{R_{2}} u_{r_{2}}^{2} y_{r_{2} j}^{2}-\sum_{i_{2}=1}^{I_{2}} v_{i_{2}}^{2} x_{i_{2} j}^{2}
$$$$
-\sum_{d_{1}=1}^{D_{1}} w_{d_{1}}^{1} z_{d_{1} j}^{1} \geq 0, \quad j=1, \ldots, n,
$$$$
\sum_{r_{3}=1}^{R_{3}} u_{r_{3}}^{3} y_{r_{3} j}^{3}-\sum_{i_{1}=1}^{I_{1}} v_{i_{3}}^{3} x_{i_{3} j}^{3}-\sum_{d_{2}=1}^{D_{2}} w_{d_{2}}^{2} z_{d_{2} j}^{2} \geq 0,
$$$$
j=1, \ldots, n,
$$$$
\sum_{d_{1}=1}^{D_{1}} w_{d_{1}}^{1} z_{d_{1} o}^{1}-\sum_{r_{1}=1}^{R_{1}} u_{r_{1}}^{1} y_{r_{1} o}^{1}-\varphi_{o}^{L *} \sum_{i_{1}=1}^{I_{1}} v_{i_{1}}^{1} x_{i_{1} o}^{1}=0,
$$$$
u_{r_{1}}^{1}, u_{r_{2}}^{2}, u_{r_{3}}^{3} \geq \varepsilon ; \quad r_{1}=1, \ldots, R_{1} ; \quad r_{2}=1, \ldots, R_{2} ;
$$$$
r_{3}=1, \ldots, R_{3} \text {, }
$$

$$
\begin{aligned}
& v_{i_{1}}^{1}, v_{i_{2}}^{2}, v_{i_{3}}^{3} \geq \varepsilon ; \quad i_{1}=1, \ldots, I_{1} ; \quad i_{2}=1, \ldots, I_{2} ; \\
& i_{3}=1, \ldots, I_{3}, \\
& w_{d_{1}}^{1}, w_{d_{2}}^{2} \geq \varepsilon ; \quad d_{1}=1, \ldots, D_{1} ; \quad d_{2}=1, \ldots, D_{2} .
\end{aligned}
$$

\section{Model 11:}

$$
\phi_{o}^{2 F *}=\min \sum_{r_{3}=1}^{R_{3}} u_{r_{3}}^{3} y_{r_{3} o}^{3}
$$

s.t.:

$$
\begin{aligned}
& \sum_{i_{1}=1}^{I_{1}} v_{i_{3}}^{3} x_{i_{3} o}^{3}+\sum_{d_{2}=1}^{D_{2}} w_{d_{2}}^{2} z_{d_{2} o}^{2}=1 \\
& \sum_{d_{1}=1}^{D_{1}} w_{d_{1}}^{1} z_{d_{1} j}^{1}-\sum_{r_{1}=1}^{R_{1}} u_{r_{1}}^{1} y_{r_{1} j}^{1}-\sum_{i_{1}=1}^{I_{1}} v_{i_{1}}^{1} x_{i_{1} j}^{1} \geq 0, \\
& \quad j=1, \ldots, n,
\end{aligned}
$$

$$
\begin{aligned}
\sum_{d_{2}=1}^{D_{2}} w_{d_{2}}^{2} z_{d_{2} j}^{2} & +\sum_{r_{2}=1}^{R_{2}} u_{r_{2}}^{2} y_{r_{2} j}^{2}-\sum_{i_{2}=1}^{I_{2}} v_{i_{2}}^{2} x_{i_{2} j}^{2} \\
& -\sum_{d_{1}=1}^{D_{1}} w_{d_{1}}^{1} z_{d_{1} j}^{1} \geq 0, \quad j=1, \ldots, n,
\end{aligned}
$$$$
\sum_{r_{3}=1}^{R_{3}} u_{r_{3}}^{3} y_{r_{3} j}^{3}-\sum_{i_{1}=1}^{I_{1}} v_{i_{3}}^{3} x_{i_{3} j}^{3}-\sum_{d_{2}=1}^{D_{2}} w_{d_{2}}^{2} z_{d_{2} j}^{2} \geq 0
$$$$
j=1, \ldots, n,
$$$$
\sum_{d_{1}=1}^{D_{1}} w_{d_{1}}^{1} z_{d_{1} o}^{1}-\sum_{r_{1}=1}^{R_{1}} u_{r_{1}}^{1} y_{r_{1} o}^{1}-\varphi_{o}^{L *} \sum_{i_{1}=1}^{I_{1}} v_{i_{1}}^{1} x_{i_{1} o}^{1}=0
$$$$
\sum_{d_{2}=1}^{D_{2}} w_{d_{2}}^{2} z_{d_{2} o}^{2}+\sum_{r_{2}=1}^{R_{2}} u_{r_{2}}^{2} y_{r_{2} o}^{2}-\varphi_{o}^{1 F *} \sum_{i_{1}=1}^{I_{1}} v_{i_{2}}^{2} x_{i_{2} o}^{2}
$$$$
-\varphi_{o}^{1 F *} \sum_{d_{1}=1}^{D_{1}} w_{d_{1}}^{1} z_{d_{1} o}^{1}=0
$$

$u_{r_{1}}^{1}, u_{r_{2}}^{2}, u_{r_{3}}^{3} \geq \varepsilon ; \quad r_{1}=1, \ldots, R_{1} ; \quad r_{2}=1, \ldots, R_{2}$

$r_{3}=1, \ldots, R_{3}$,

$v_{i_{1}}^{1}, v_{i_{2}}^{2}, v_{i_{3}}^{3} \geq \varepsilon ; \quad i_{1}=1, \ldots, I_{1} ; \quad i_{2}=1, \ldots, I_{2}$

$i_{3}=1, \ldots, I_{3} ; \quad j=1, \ldots, n$,

$w_{d_{1}}^{1}, w_{d_{2}}^{2} \geq \varepsilon ; \quad d_{1}=1, \ldots, D_{1} ; \quad d_{2}=1, \ldots, D_{2}$. 
It must be observed that the stages of Figure 1 are in series, based on the views of Kao and Hwang [53], and a multiplicative approach is used to compute overall efficiency. Hence, by utilizing the results of the abovementioned Models 6-11, the maximum overall optimistic efficiency $\left(\theta_{o}^{\text {overall }}\right)$, the minimum overall pessimistic efficiency ( $\left.\varphi_{o}^{\text {overall }}\right)$ as well as the overall efficiency based on the double-frontier ( $\left.\phi_{o}^{\text {overall }}\right)$, are defined, respectively, as follows:

$$
\begin{aligned}
& \theta_{o}^{\text {overall }}=\theta_{o}^{L *} \cdot \theta_{o}^{1 F *} \cdot \theta_{o}^{2 F *}, \quad \varphi_{o}^{\text {overall }}=\varphi_{o}^{L *} \cdot \varphi_{o}^{1 F *} \cdot \varphi_{o}^{2 F *}, \\
& \phi_{o}^{\text {overall }}=\sqrt{\theta_{o}^{\text {overall }} \cdot \varphi_{o}^{\text {overall }} .}
\end{aligned}
$$

\subsubsection{Second scenario}

In the second scenario, The first stage as the leader and the second and third stages together are depicted in the form of a follower. On these bases, the efficiency of the optimistic leader stage is illustrated as $\theta_{o}^{L}$, whereas, the optimistic efficiencies of the second and third stages are shown as $\theta_{o}^{1 F}$ and $\theta_{o}^{2 F}$ respectively; and the optimistic efficiency of the second and third stages together is demonstrated as $\theta_{o}^{12 F}$. In fact, the difference between this scenario and the first scenario lies in the role of the second and third stages. Therefore, the maximal optimistic efficiency of the leader stage $\left(\theta_{o}^{L *}\right)$ and the minimal pessimistic efficiency of the leader stage $\left(\varphi_{o}^{L *}\right)$ are brought to hand, respectively, from Models 6 and 9 in the first scenario. Similarly, the efficiencies of the second and third stages are hybrid, being attentive to the fact that they are in series and are defined as $\theta_{o}^{12 F}=\theta_{o}^{1 F} \cdot \theta_{o}^{2 F}$, according to the work of Kao and Hwang [53]. Hence, the maximal efficiency for the follower stage $\left(\theta_{o}^{12 F}\right)$ from the optimistic viewpoint is found and rendered as shown beneath.

\section{Model 12:}

$$
\begin{aligned}
\theta_{o}^{12 F *}= & \max \frac{\sum_{d_{2}=1}^{D_{2}} w_{d_{2}}^{2} z_{d_{2} o}^{2}+\sum_{r_{2}=1}^{R_{2}} u_{r_{2}}^{2} y_{r_{2} o}^{2}}{\sum_{i_{2}=1}^{I_{2}} v_{i_{2}}^{2} x_{i_{2} o}^{2}+\sum_{d_{1}=1}^{D_{1}} w_{d_{1}}^{1} z_{d_{1} o}^{1}} \\
& \frac{\sum_{r_{3}=1}^{R_{3}} u_{r_{3}}^{3} y_{r_{3} o}^{3}}{\sum_{i_{3}=1}^{I_{3}} v_{i_{3}}^{3} x_{i_{3} o}^{3}+\sum_{d_{2}=1}^{D_{2}} w_{d_{2}}^{2} z_{d_{2} o}^{2}}
\end{aligned}
$$

s.t.:

$$
\begin{aligned}
& \frac{\sum_{d_{1}=1}^{D_{1}} w_{d_{1}}^{1} z_{d_{1} j}^{1}-\sum_{r_{1}=1}^{R_{1}} u_{r_{1}}^{1} y_{r_{1} j}^{1}}{\sum_{i_{1}=1}^{I_{1}} v_{i_{1}}^{1} x_{i_{1} j}^{1}} \leq 1, \quad j=1, \ldots, n, \\
& \frac{\sum_{d_{2}=1}^{D_{2}} w_{d_{2}}^{2} z_{d_{2} j}^{2}+\sum_{r_{2}=1}^{R_{2}} u_{r_{2}}^{2} y_{r_{2} j}^{2}}{\sum_{i_{2}=1}^{I_{2}} v_{i_{2}}^{2} x_{i_{2} j}^{2}+\sum_{d_{1}=1}^{D_{1}} w_{d_{1}}^{1} z_{d_{1} j}^{1}} \leq 1, \quad j=1, \ldots, n, \\
& \frac{\sum_{r_{3}=1}^{R_{3}} u_{r_{3}}^{3} y_{r_{3} j}^{3}}{\sum_{i_{3}=1}^{I_{3}} v_{i_{3}}^{3} x_{i_{3} j}^{3}+\sum_{d_{2}=1}^{D_{2}} w_{d_{2}}^{2} z_{d_{2} j}^{2}} \leq 1, \quad j=1, \ldots, n,
\end{aligned}
$$

$$
\begin{aligned}
& \frac{\sum_{d_{1}=1}^{D_{1}} w_{d_{1}}^{1} z_{d_{1} o}^{1}-\sum_{r_{1}=1}^{R_{1}} u_{r_{1}}^{1} y_{r_{1} o}^{1}}{\sum_{i_{1}=1}^{I_{1}} v_{i_{1}}^{1} x_{i_{1} o}^{1}}=\theta_{o}^{L *}, \\
& u_{r_{1}}^{1}, u_{r_{2}}^{2}, u_{r_{3}}^{3} \geq \varepsilon ; \quad r_{1}=1, \ldots, R_{1} ; \quad r_{2}=1, \ldots, R_{2} ; \\
& r_{3}=1, \ldots, R_{3}, \\
& v_{i_{1}}^{1}, v_{i_{2}}^{2}, v_{i_{3}}^{3} \geq \varepsilon ; \quad i_{1}=1, \ldots, I_{1} ; \quad i_{2}=1, \ldots, I_{2} ; \\
& i_{3}=1, \ldots, I_{3}, \\
& w_{d_{1}}^{1}, w_{d_{2}}^{2} \geq \varepsilon ; \quad d_{1}=1, \ldots, D_{1} ; \quad d_{2}=1, \ldots, D_{2} .
\end{aligned}
$$

The maximum and overall efficiency of the follower stage $\left(\theta_{o}^{12 F}\right)$ is gained by Model 12 , on condition that the efficiencies of the other stages are less than 1; and, according to the approach of Li et al., the efficiency of the leader stage remain constant. Based on the work of Wang et al. [51], Model 12 is described, as given below, in order to attain the minimum efficiency of the follower stage $\left(\varphi_{o}^{12 F}\right)$ from the pessimistic viewpoint.

\section{Model 13:}

$$
\begin{aligned}
\varphi_{o}^{12 F *}= & \min \frac{\sum_{d_{2}=1}^{D_{2}} w_{d_{2}}^{2} z_{d_{2} o}^{2}+\sum_{r_{2}=1}^{R_{2}} u_{r_{2}}^{2} y_{r_{2} o}^{2}}{\sum_{i_{2}=1}^{I_{2}} v_{i_{2}}^{2} x_{i_{2} o}^{2}+\sum_{d_{1}=1}^{D_{1}} w_{d_{1}}^{1} z_{d_{1} o}^{1}} \\
& \cdot \frac{\sum_{r_{3}=1}^{R_{3}} u_{r_{3}}^{3} y_{r_{3} o}^{3}}{\sum_{i_{3}=1}^{I_{3}} v_{i_{3}}^{3} x_{i_{3} o}^{3}+\sum_{d_{2}=1}^{D_{2}} w_{d_{2}}^{2} z_{d_{2} o}^{2}}
\end{aligned}
$$

s.t.:

$\frac{\sum_{d_{1}=1}^{D_{1}} w_{d_{1}}^{1} z_{d_{j} j}^{1}-\sum_{r_{1}=1}^{R_{1}} u_{r_{1}}^{1} y_{r_{1} j}^{1}}{\sum_{i_{1}=1}^{I_{1}} v_{i_{1}}^{1} x_{i_{1} j}^{1}} \geq 1, \quad j=1, \ldots, n$,

$\frac{\sum_{d_{2}=1}^{D_{2}} w_{d_{2}}^{2} z_{d_{2 j}}^{2}+\sum_{r_{2}=1}^{R_{2}} u_{r_{2}}^{2} y_{r_{2} j}^{2}}{\sum_{i_{2}=1}^{I_{2}} v_{i_{2}}^{2} x_{i_{2} j}^{2}+\sum_{d_{1}=1}^{D_{1}} w_{d_{1}}^{1} z_{d_{1} j}^{1}} \geq 1, \quad j=1, \ldots, n$,

$\frac{\sum_{r_{3}=1}^{R_{3}} u_{r_{3}}^{3} y_{r_{3} j}^{3}}{\sum_{i_{3}=1}^{I_{3}} v_{i_{3}}^{3} x_{i_{3} j}^{3}+\sum_{d_{2}=1}^{D_{2}} w_{d_{2}}^{2} z_{d_{2} j}^{2}} \geq 1, \quad j=1, \ldots, n$,

$\frac{\sum_{d_{1}=1}^{D_{1}} w_{d_{1}}^{1} z_{d_{1} o}^{1}-\sum_{r_{1}=1}^{R_{1}} u_{r_{1}}^{1} y_{r_{1} o}^{1}}{\sum_{i_{1}=1}^{I_{1}} v_{i_{1}}^{1} x_{i_{1} o}^{1}}=\varphi_{o}^{L *}$,

$u_{r_{1}}^{1}, u_{r_{2}}^{2}, u_{r_{3}}^{3} \geq \varepsilon ; \quad r_{1}=1, \ldots, R_{1} ; \quad r_{2}=1, \ldots, R_{2}$

$r_{3}=1, \ldots, R_{3}$,

$v_{i_{1}}^{1}, v_{i_{2}}^{2}, v_{i_{3}}^{3} \geq \varepsilon ; \quad i_{1}=1, \ldots, I_{1} ; \quad i_{2}=1, \ldots, I_{2} ;$

$i_{3}=1, \ldots, I_{3}$,

$w_{d_{1}}^{1}, w_{d_{2}}^{2} \geq \varepsilon ; \quad d_{1}=1, \ldots, D_{1} ; \quad d_{2}=1, \ldots, D_{2}$. 
Models 12 and 13 are nonlinear, and in the third section of this paper, an innovative approach in resolving them is utilized. By assuming that Models 12 and 13 are solved and given that the stages are in series (Figure $1)$, total and maximal optimistic efficiency ( $\theta_{o}^{\text {overall }}$ ) is defined, minimal and total pessimistic efficiency $\left(\varphi_{0}^{\text {overall }}\right)$ and overall efficiency with the double-frontier $\left(\phi_{o}^{\text {overall }}\right)$, which are, respectively, specified below:

$$
\begin{aligned}
& \theta_{o}^{\text {overall }}=\theta_{o}^{L *} \cdot \theta_{o}^{12 F *}, \quad \varphi_{o}^{\text {overall }}=\varphi_{o}^{L *} \cdot \varphi_{o}^{12 F *} \\
& \phi_{o}^{\text {overall }}=\sqrt{\theta_{o}^{\text {overall }} \cdot \varphi_{o}^{\text {overall }}} .
\end{aligned}
$$

\subsubsection{Third scenario}

In this scenario, which is comprised of the proposed approach of this paper, efforts have been made to insert the goals of the managers into the models. In the third scenario, the first stage is designated as the "leader" and it is assumed that the second and third stages together, are in the form of a "follower". The dissimilarity between this scenario and the second scenario is that in such circumstances, collaboration between the leader and followers is taken into consideration. In the second scenario, the leader stage concentrates only on maximizing its efficiency, which causes deterioration in the efficiency of the followers. In this scenario and under such circumstances, the leader optimizes its efficiency, so that the efficiency of the followers does not reduce from a certain level, or, in fact, the leader maximizes its efficiency to forestall the eradication of the followers. Actually, the leaderfollower characteristic is a non-cooperative game, which is hybrid with a cooperative approach in this section. In accordance with this, the maximal efficiency of the leader stage is described from the optimistic viewpoint as shown beneath.

\section{Model 14:}

$$
\begin{gathered}
\theta_{o}^{L *}=\max \left\{\theta_{o}^{L} \mid \theta_{o}^{1 F} \geq c_{1}, \quad \theta_{o}^{2 F} \geq c_{2}, \quad \theta_{j}^{L} \leq 1,\right. \\
\left.\theta_{j}^{1 F} \leq 1, \quad \theta_{j}^{2 F} \leq 1, \quad j=1, \ldots, n\right\} .
\end{gathered}
$$

All variables in Model 14 are non-negative. Model 14 secures the maximal efficiency of the leader stage, on condition that the efficiency of the other stages are less than 1 and for $D M U_{o}$, the efficiency of the second and third stages is not lower than the values of $c_{1}$ and $c_{2}$, respectively. The values of $c_{1}$ and $c_{2}$ are actually the minimal efficiencies of the second and third stages which are numerals at intervals of (0 and 1), in accordance with the goals of managers. It should be noted that if the values of $c_{1}=c_{2}=\varepsilon$ are considered such that they are closer to $(0)$, then, the two constraints $\theta_{o}^{1 F} \geq c_{1}, \theta_{o}^{2 F} \geq c_{2}$ are simply redundant. So, Model 14 is feasible. But there could be a possibility that in reality, the goals of managers are not capable of being attained and the model becomes superfluous. Hence, the concept of 'goal programming' is utilized and the two assigned values, $\alpha_{1}, \alpha_{2}$, are reduced $\left(\theta_{o}^{1 F} \geq c_{1}-\alpha_{1}, \theta_{o}^{2 F} \geq c_{2}-\alpha_{2}\right)$ from the opinion of managers under contemplation, so that by using Model 15, conditions for securing the goal of the managers is surveyed. Model 14 is a fractional model and by utilizing the Charnes-Cooper conversion (1962), as well as contemplating the GP concept, as illustrated beneath, it is converted into a linear model:

\section{Model 15:}

$$
\theta_{o}^{L *}=\max \sum_{d_{1}=1}^{D_{1}} w_{d_{1}}^{1} z_{d_{1} o}^{1}-\sum_{r_{1}=1}^{R_{1}} u_{r_{1}}^{1} y_{r_{1} o}^{1}-M\left(\alpha_{1}+\alpha_{2}\right),
$$

s.t.:

$$
\begin{aligned}
& \sum_{i_{1}=1}^{I_{1}} v_{i_{1}}^{1} x_{i_{1} o}^{1}=1 \\
& \sum_{d_{1}=1}^{D_{1}} w_{d_{1}}^{1} z_{d_{1} j}^{1}-\sum_{r_{1}=1}^{R_{1}} u_{r_{1}}^{1} y_{r_{1} j}^{1}-\sum_{i_{1}=1}^{I_{1}} v_{i_{1}}^{1} x_{i_{1} j}^{1} \leq 0, \\
& j=1, \ldots, n, \\
& \sum_{d_{2}=1}^{D_{2}} w_{d_{2}}^{2} z_{d_{2} j}^{2}+\sum_{r_{2}=1}^{R_{2}} u_{r_{2}}^{2} y_{r_{2} j}^{2}-\sum_{i_{2}=1}^{I_{2}} v_{i_{2}}^{2} x_{i_{2} j}^{2} \\
& -\sum_{d_{1}=1}^{D_{1}} w_{d_{1}}^{1} z_{d_{1} j}^{1} \leq 0, \quad j=1, \ldots, n,
\end{aligned}
$$

$$
\begin{gathered}
\sum_{r_{3}=1}^{R_{3}} u_{r_{3}}^{3} y_{r_{3} j}^{3}-\sum_{i_{3}=1}^{I_{3}} v_{i_{3}}^{3} x_{i_{3} j}^{3}-\sum_{d_{2}=1}^{D_{2}} w_{d_{2}}^{2} z_{d_{2} j}^{2} \leq 0 \\
j=1, \ldots, n
\end{gathered}
$$$$
\left(c_{1}-\alpha_{1}\right) \sum_{d_{1}=1}^{D_{1}} w_{d_{1}}^{1} z_{d_{1} o}^{1}+\left(c_{1}-\alpha_{1}\right) \sum_{i_{2}=1}^{I_{2}} v_{i_{2}}^{2} x_{i_{2} o}^{2}
$$$$
-\sum_{d_{2}=1}^{D_{2}} w_{d_{2}}^{2} z_{d_{2} o}^{2}-\sum_{r_{2}=1}^{R_{2}} u_{r_{2}}^{2} y_{r_{2} o}^{2} \leq 0
$$$$
\left(c_{2}-\alpha_{2}\right) \sum_{i_{3}=1}^{I_{3}} v_{i_{3}}^{3} x_{i_{3 \circ}}^{3}+\left(c_{2}-\alpha_{2}\right) \sum_{d_{2}=1}^{D_{2}} w_{d_{2}}^{2} z_{d_{2} o}^{2}
$$$$
-\sum_{r_{3}=1}^{R_{3}} u_{r_{3}}^{3} y_{r_{3} o}^{3} \leq 0
$$ 


$$
\begin{aligned}
& u_{r_{1}}^{1}, u_{r_{2}}^{2}, u_{r_{3}}^{3} \geq \varepsilon ; \quad r_{1}=1, \ldots, R_{1} ; \quad r_{2}=1, \ldots, R_{2} ; \\
& r_{3}=1, \ldots, R_{3} \\
& v_{i_{1}}^{1}, v_{i_{2}}^{2}, v_{i_{3}}^{3} \geq \varepsilon ; \quad i_{1}=1, \ldots, I_{1} ; \quad i_{2}=1, \ldots, I_{2} ; \\
& i_{3}=1, \ldots, I_{3}, \\
& w_{d_{1}}^{1}, w_{d_{2}}^{2} \geq \varepsilon ; \quad d_{1}=1, \ldots, D_{1} ; \quad d_{2}=1, \ldots, D_{2} .
\end{aligned}
$$

In Model 15 " $M$ " denotes a large numeral, which factually is a penalty that causes the manager goal to be achievable. It should be mentioned that in the case where, $\alpha_{1}=0, \alpha_{2}=0$, Model 15 is feasible from the point of the manager goal. If this is not the issue, the manager is requested to reduce his goals $\left(c_{1}, c_{2}\right)$ to the measurement of $\alpha_{1}$ and $\alpha_{2}$ to make the model possible. On the basis of the work of Wang et al. [51], Model 15 is modified, as shown beneath, to obtain the efficiency of the leader stage from the pessimistic view. Similar to the optimistic approach, the pessimistic efficiency of the leader stage is obtained, under conditions where the follower stages are at a distance from the inefficient frontier, i.e. $\varphi_{o}^{1 F} \geq c_{3}-\alpha_{3}, \varphi_{o}^{2 F} \geq c_{4}-\alpha_{4}$, in which $c_{3}, c_{4} \geq 1$.

\section{Model 16:}

$$
\varphi_{o}^{L *}=\min \sum_{d_{1}=1}^{D_{1}} w_{d_{1}}^{1} z_{d_{1} o}^{1}-\sum_{r_{1}=1}^{R_{1}} u_{r_{1}}^{1} y_{r_{1} o}^{1}+M\left(\alpha_{3}+\alpha_{4}\right)
$$

s.t.:

$$
\begin{aligned}
& \sum_{i_{1}=1}^{I_{1}} v_{i_{1}}^{1} x_{i_{1} o}^{1}=1 \\
& \sum_{d_{1}=1}^{D_{1}} w_{d_{1}}^{1} z_{d_{1} j}^{1}-\sum_{r_{1}=1}^{R_{1}} u_{r_{1}}^{1} y_{r_{1} j}^{1}-\sum_{i_{1}=1}^{I_{1}} v_{i_{1}}^{1} x_{i_{1} j}^{1} \geq 0, \\
& j=1, \ldots, n, \\
& \sum_{d_{2}=1}^{D_{2}} w_{d_{2}}^{2} z_{d_{2} j}^{2}+\sum_{r_{2}=1}^{R_{2}} u_{r_{2}}^{2} y_{r_{2} j}^{2}-\sum_{i_{2}=1}^{I_{2}} v_{i_{2}}^{2} x_{i_{2} j}^{2} \\
& \quad-\sum_{d_{1}=1}^{D_{1}} w_{d_{1}}^{1} z_{d_{1} j}^{1} \geq 0, j=1, \ldots, n, \\
& \sum_{r_{3}=1}^{R_{3}} u_{r_{3}}^{3} y_{r_{3} j}^{3}-\sum_{i_{3}=1}^{I_{3}} v_{i_{3}}^{3} x_{i_{3} j}^{3}-\sum_{d_{2}=1}^{D_{2}} w_{d_{2}}^{2} z_{d_{2} j}^{2} \geq 0, \\
& j=1, \ldots, n,
\end{aligned}
$$

$$
\begin{aligned}
& \sum_{d_{2}=1}^{D_{2}} w_{d_{2}}^{2} z_{d_{2} o}^{2}+\sum_{r_{2}=1}^{R_{2}} u_{r_{2}}^{2} y_{r_{2} o}^{2}-\left(c_{3}-\alpha_{3}\right) \sum_{d_{1}=1}^{D_{1}} w_{d_{1}}^{1} z_{d_{1} o}^{1} \\
& -\left(c_{3}-\alpha_{3}\right) \sum_{i_{2}=1}^{I_{2}} v_{i_{2}}^{2} x_{i_{2} o}^{2} \geq 0 \\
& \sum_{r_{3}=1}^{R_{3}} u_{r_{3}}^{3} y_{r_{3} o}^{3}-\left(c_{4}-\alpha_{4}\right) \sum_{i_{3}=1}^{I_{3}} v_{i_{3}}^{3} x_{i_{3} o}^{3}-\left(c_{4}-\alpha_{4}\right) \\
& \sum_{d_{2}=1}^{D_{2}} w_{d_{2}}^{2} z_{d_{2} o}^{2} \geq 0 \\
& u_{r_{1}}^{1}, u_{r_{2}}^{2}, u_{r_{3}}^{3} \geq \varepsilon ; \quad r_{1}=1, \ldots, R_{1} ; \quad r_{2}=1, \ldots, R_{2} \\
& r_{3}=1, \ldots, R_{3}, \\
& v_{i_{1}}^{1}, v_{i_{2}}^{2}, v_{i_{3}}^{3} \geq \varepsilon ; \quad i_{1}=1, \ldots, I_{1} ; \quad i_{2}=1, \ldots, I_{2} \\
& i_{3}=1, \ldots, I_{3}, \\
& w_{d_{1}}^{1}, w_{d_{2}}^{2} \geq \varepsilon ; \quad d_{1}=1, \ldots, D_{1} ; \quad d_{2}=1, \ldots, D_{2} .
\end{aligned}
$$

The optimistic approach of " $M$ " that is a large numerical, which under this circumstance and with due attention to the type of objective function, has been supplemented to the model, in order to fulfill the manager goal. It should be observed that in the case where $\alpha_{1}=0, \alpha_{2}=0$ Model 16 is feasible, in respect to the opinion of the manager, or else, the manager is requested to reduce his goals $\left(c_{3}, c_{4}\right)$ to the measurement of $a_{3}, a_{4}$ to make the model possible.

To compute maximal efficiency for the follower stage $\left(\theta_{o}^{12 F}\right)$ from the optimistic viewpoint, and minimal efficiency for the follower stage $\left(\varphi_{o}^{12 F}\right)$ from the pessimistic viewpoint, the operation will be according to the second scenario; and the second and third stages will be assumed as one stage and the efficiency of the follower stage will be obtained on the basis of Models 12 and 13. It should be observed that, under such conditions, the efficiency values of the leader stage $\left(\theta_{o}^{L^{*}}\right.$ and $\varphi_{o}^{L^{*}}$ ) are found in Models 12 and 13 on the basis of the results of Models 15 and 16. Likewise, two constraints $\theta_{o}^{1 F} \geq c_{1}-\alpha_{1}$ and $\theta_{o}^{2 F} \geq c_{2}-\alpha_{2}$ are supplemented to Model 12, including two constraints $\varphi_{o}^{1 F} \geq c_{3}-\alpha_{3}$ and $\varphi_{o}^{2 F} \geq c_{4}-\alpha_{4}$ which are added to Model 13. The values of $\alpha_{i}, i=1,2,3,4$ for these 4 constraints are obtained from Models 15 and 16 . Finally, by utilizing Eqs. (16) in this scenario, maximal overall optimistic efficiency, minimal overall pessimistic efficiency and total efficiency relative to the doublefrontier are attained. 


\section{Heuristic approach to solve nonlinear models}

Two exploratory approaches are proposed for optimistic and pessimistic views relatively. For the first time, an exploratory approach has been developed in regard to the pessimistic perspective or condition. To the best of our knowledge, this modeling has not been performed, as to this conceptual approach under pessimistic conditions, until now. Similarly, an exploratory approach has been implemented from the optimistic standpoint, $r$, relative to the leader-follower concept. Thereby, the exploratory approach from the pessimistic angle is proposed in this paper. In this section, a solution will be used to gain the efficiencies of the follower $\left(\theta_{o}^{12 F}, \varphi_{o}^{12 F}\right)$ in the second and third scenarios, which will be described in accordance to the second scenario. Due to the presence of additional inputs and outputs in the first, second and third stages, Models 12 and 13 are nonlinear. To solve these models a heuristic approach is used, as described in the following.

\subsection{A heuristic method from optimistic viewpoint}

It is known that the objective function of Model 12 is a multiplicative efficiency of the two-stages, i.e. $\theta_{o}^{12 F *}=$ $\max \theta_{o}^{1 F} \cdot \theta_{o}^{2 F}$. Let us consider the first stage $\left(\theta_{o}^{1 F}\right)$ as a variable that changes between interval $\left[0, \theta_{o}^{1 F-\max }\right]$. By using Eq. (20), this variable can be moved into its interval:

$$
\begin{aligned}
& \theta_{o}^{1 F}=\theta_{o}^{1 F-\max }-k_{1} \Delta \varepsilon \\
& k_{1}=0,1, \ldots,\left[\frac{\theta_{o}^{1 F-\max }}{\Delta \varepsilon}\right]+1 .
\end{aligned}
$$

In taking $\Delta \varepsilon$ as a step size, it is considered an extremely small amount and $\theta_{o}^{1 F-\max }$ is described as the maximum efficiency of the first follower stage. Its value is capable of being computed by the model below:

\section{Model 17:}

$$
\begin{gathered}
\theta_{o}^{1 F-\max }=\max \left\{\theta_{o}^{1 F} \mid \theta_{j}^{L} \leq 1, \quad \theta_{j}^{1 F} \leq 1, \quad \theta_{j}^{2 F} \leq 1,\right. \\
j=1, \ldots, n\} .
\end{gathered}
$$

Model 17 secures the maximum efficiency of the first follower stage, under conditions where the efficiencies of the other stages are less than 1. In actual fact, this model, irrespective of leader-follower correlations, attributes the highest efficiency to the second stage. This model is a fractional model and by utilizing the Charnes-Cooper conversion (1962), as illustrated beneath, it is converted into a linear model.
Model 18:

$$
\theta_{o}^{1 F-\max }=\max \sum_{d_{2}=1}^{D_{2}} w_{d_{2}}^{2} z_{d_{2} o}^{2}+\sum_{r_{2}=1}^{R_{2}} u_{r_{2}}^{2} y_{r_{2} o}^{2}
$$

s.t.:

$$
\begin{aligned}
& \sum_{i_{1}=1}^{I_{1}} v_{i_{2}}^{2} x_{i_{2} o}^{2}+\sum_{d_{1}=1}^{D_{1}} w_{d_{1}}^{1} z_{d_{1} o}^{1}=1, \\
& \sum_{d_{1}=1}^{D_{1}} w_{d_{1}}^{1} z_{d_{1} j}^{1}-\sum_{r_{1}=1}^{R_{1}} u_{r_{1}}^{1} y_{r_{1} j}^{1}-\sum_{i_{1}=1}^{I_{1}} v_{i_{1}}^{1} x_{i_{1} j}^{1} \leq 0, \\
& j=1, \ldots, n, \\
& \sum_{d_{2}=1}^{D_{2}} w_{d_{2}}^{2} z_{d_{2} j}^{2}+\sum_{r_{2}=1}^{R_{2}} u_{r_{2}}^{2} y_{r_{2} j}^{2}-\sum_{i_{2}=1}^{I_{2}} v_{i_{2}}^{2} x_{i_{2} j}^{2} \\
& \quad-\sum_{d_{1}=1}^{D_{1}} w_{d_{1}}^{1} z_{d_{1} j}^{1} \leq 0, j=1, \ldots, n, \\
& \sum_{d_{1}}^{1}, w_{d_{2}}^{2} \geq \varepsilon ; \quad d_{1}=1, \ldots, D_{1} ; d_{2}=1, \ldots, D_{2} . \quad \\
& \sum_{r_{3}=1}^{R_{3}} u_{r_{3}}^{3} y_{r_{3} j}^{3}-\sum_{i_{3}=1}^{I_{3}} v_{i_{3}}^{3} x_{i_{3} j}^{3}-\sum_{d_{2}=1}^{D_{2}} w_{d_{2}}^{2} z_{d_{2} j}^{2} \leq 0, \\
& v_{i_{1}}^{1}, v_{i_{2}}^{2}, v_{i_{3}}^{3} \geq \varepsilon ; i_{1}=1, \ldots, I_{1} ; i_{2}=1, \ldots, I_{2} ; \\
& u_{r_{1}}^{1}, u_{r_{2}}^{2}, u_{r_{3}}^{3} \geq \varepsilon ; r_{1}=1, \ldots, R_{1} ; r_{2}=1, \ldots, R_{2} ; \\
& r_{3}=1, \ldots, R_{3},
\end{aligned}
$$

In determining the value of $\theta_{o}^{1 F-\max }$ by Model 18 , Model 12 is converted into the following model.

Model 19:

$$
\begin{gathered}
\theta_{o}^{12 F *}=\max \left\{\theta_{o}^{1 F} \theta_{o}^{2 F} \mid \theta_{j}^{L} \leq 1, \quad \theta_{j}^{1 F} \leq 1, \quad \theta_{j}^{2 F} \leq 1\right. \\
\theta_{o}^{L}=\theta_{o}^{L *}, \quad \theta_{o}^{1 F}=\frac{O_{o}^{2}}{I_{o}^{2}}, \quad \theta_{o}^{1 F} \in\left[0, \theta_{o}^{1 F-\max }\right], \\
j=1, \ldots, n\} .
\end{gathered}
$$

In Model 19, $\theta_{o}^{1 F}$ was considered in the objective function as a variable and the constraint which specified this variable, together with its interval of modification, 
was added to the model. In this model, the efficiency of the second stage or $\theta_{o}^{1 F}$ has been demonstrated briefly in the form of output to input. The mentioned model is a fractional one and by utilizing the Charnes-Cooper conversion (1962), as illustrated below, it is converted into a linear model:

\section{Model 20:}

$$
\theta_{o}^{12 F *}=\max \theta_{o}^{1 F} \cdot \sum_{r_{3}=1}^{R_{3}} u_{r_{3}}^{3} y_{r_{3} o}^{3},
$$

s.t.:

$$
\begin{aligned}
& \sum_{i_{3}=1}^{I_{3}} v_{i_{3}}^{3} x_{i_{3} o}^{3}+\sum_{d_{2}=1}^{D_{2}} w_{d_{2}}^{2} z_{d_{2} o}^{2}=1, \\
& \sum_{d_{1}=1}^{D_{1}} w_{d_{1}}^{1} z_{d_{1} j}^{1}-\sum_{r_{1}=1}^{R_{1}} u_{r_{1}}^{1} y_{r_{1} j}^{1}-\sum_{i_{1}=1}^{I_{1}} v_{i_{1}}^{1} x_{i_{1} j}^{1} \leq 0, \\
& j=1, \ldots, n, \\
& \sum_{d_{2}=1}^{D_{2}} w_{d_{2}}^{2} z_{d_{2} j}^{2}+\sum_{r_{2}=1}^{R_{2}} u_{r_{2}}^{2} y_{r_{2} j}^{2}-\sum_{i_{2}=1}^{I_{2}} v_{i_{2}}^{2} x_{i_{2} j}^{2} \\
& -\sum_{d_{1}=1}^{D_{1}} w_{d_{1}}^{1} z_{d_{1} j}^{1} \leq 0, \quad j=1, \ldots, n, \\
& \sum_{r_{3}=1}^{R_{3}} u_{r_{3}}^{3} y_{r_{3} j}^{3}-\sum_{i_{3}=1}^{I_{3}} v_{i_{3}}^{3} x_{i_{3} j}^{3}-\sum_{d_{2}=1}^{D_{2}} w_{d_{2}}^{2} z_{d_{2} j}^{2} \leq 0 \\
& j=1, \ldots, n, \\
& \sum_{d_{1}=1}^{D_{1}} w_{d_{1}}^{1} z_{d_{1} o}^{1}-\sum_{r_{1}=1}^{R_{1}} u_{r_{1}}^{1} y_{r_{1} o}^{1}-\theta_{o}^{L *} \sum_{i_{1}=1}^{I_{1}} v_{i_{1}}^{1} x_{i_{1} o}^{1}=0, \\
& \sum_{d_{2}=1}^{D_{2}} w_{d_{2}}^{2} z_{d_{2} o}^{2}+\sum_{r_{2}=1}^{R_{2}} u_{r_{2}}^{2} y_{r_{2} o}^{2}-\theta_{o}^{1 F *} \\
& \left(\sum_{i_{2}=1}^{I_{2}} v_{i_{2}}^{2} x_{i_{2} o}^{2}+\sum_{d_{1}=1}^{D_{1}} w_{d_{1}}^{1} z_{d_{1} o}^{1}\right)=0, \\
& \theta_{o}^{1 F} \in\left[0, \theta_{o}^{1 F-\max }\right] \\
& u_{r_{1}}^{1}, u_{r_{2}}^{2}, u_{r_{3}}^{3} \geq \varepsilon ; r_{1}=1, \ldots, R_{1} ; \quad r_{2}=1, \ldots, R_{2} ; \\
& r_{3}=1, \ldots, R_{3}, \\
& v_{i_{1}}^{1}, v_{i_{2}}^{2}, v_{i_{3}}^{3} \geq \varepsilon ; \quad i_{1}=1, \ldots, I_{1} ; \quad i_{2}=1, \ldots, I_{2} ; \\
& i_{3}=1, \ldots, I_{3} \text {, }
\end{aligned}
$$

$$
w_{d_{1}}^{1}, w_{d_{2}}^{2} \geq \varepsilon ; \quad d_{1}=1, \ldots, D_{1} ; \quad d_{2}=1, \ldots, D_{2} .
$$

In Model 20 and by utilizing Eq. (20), the value of $k_{1}$ is increased from 0 to its higher level, in order to solve the new model each time with $\theta_{o}^{1 F}$. For the entire conditions of $k_{1}$, Model 20 is solved and the responses of the model are assigned as $\theta_{o}^{12 F}\left(k_{1}\right)$. By comparing all the values of $\theta_{o}^{12 F}\left(k_{1}\right), \theta_{o}^{12 F^{*}}$ is defined as the maximal efficiency of the follower stage from the optimistic view. It should be noted that this proposed approach has been tested under two conditions; each time a stage has been considered as a variable. Given that the efficiency of a stage is somewhat unique, the results of these two methods have been obtained with an extremely good approximation. In order to explain this approach, one of these two conditions above have been denoted.

\subsection{A heuristic method from a pessimistic viewpoint}

It is known that the objective function of Model 13 is the multiplicative efficiency of two stages, i.e. $\varphi_{o}^{12 F *}=$ $\min \varphi_{o}^{1 F} \dot{\varphi}_{o}^{2 F}$. Similar to the optimistic view presented, $\varphi_{o}^{1 F}$ is taken as a variable in the objective function that modifies between the $\left[\varphi_{o}^{1 F-\min }, M\right]$ interval. $\varphi_{o}^{1 F}$ is described as shown below so that it can be moved within the interval:

$$
\begin{aligned}
& \varphi_{o}^{1 F}=\phi_{o}^{1 F-\min }+k_{1} \Delta \varepsilon \\
& k_{1}=0,1, \ldots,\left[\frac{M-\varphi_{o}^{1 F-\min }}{\Delta \varepsilon}\right]+1,
\end{aligned}
$$

" $M$ " is considered to be a large amount and alike the optimistic approach, $\Delta \varepsilon$ is a step size and an extremely small amount. $\varphi_{o}^{1 F-\min }$ is described as the minimum efficiency of the first follower stage and its sum can be computed by the following model.

\section{Model 21:}

$$
\begin{gathered}
\varphi_{o}^{1 F-\min }=\min \left\{\varphi_{o}^{1 F} \mid \varphi_{j}^{L} \geq 1, \quad \varphi_{j}^{1 F} \geq 1, \quad \varphi_{j}^{2 F} \geq 1\right. \\
j=1, \ldots, n\} .
\end{gathered}
$$

Model 21, secures the minimum efficiency of the first follower stage, under the condition where the efficiency of the other stages is more than 1 . In fact, this model, regardless of the leader-follower correlation, attributes the least amount of efficiency to the second stage. This model is a fractional model and by employing the Charnes-Cooper conversion (1962), it is converted into a linear model, as given below. 
Model 22:

$$
\varphi_{o}^{1 F-\min }=\min \sum_{d_{2}=1}^{D_{2}} w_{d_{2}}^{2} z_{d_{2} o}^{2}+\sum_{r_{2}=1}^{R_{2}} u_{r_{2}}^{2} y_{r_{2} o}^{2}
$$

s.t.:

$$
\begin{aligned}
& \sum_{i_{2}=1}^{I_{2}} v_{i_{2}}^{2} x_{i_{2} o}^{2}+\sum_{d_{1}=1}^{D_{1}} w_{d_{1}}^{1} z_{d_{1} o}^{1}=1, \\
& \sum_{d_{1}=1}^{D_{1}} w_{d_{1}}^{1} z_{d_{1} j}^{1}-\sum_{r_{1}=1}^{R_{1}} u_{r_{1}}^{1} y_{r_{1} j}^{1}-\sum_{i_{1}=1}^{I_{1}} v_{i_{1}}^{1} x_{i_{1} j}^{1} \geq 0, \\
& j=1, \ldots, n, \\
& \sum_{d_{2}=1}^{D_{2}} w_{d_{2}}^{2} z_{d_{2} j}^{2}+\sum_{r_{2}=1}^{R_{2}} u_{r_{2}}^{2} y_{r_{2} j}^{2}-\sum_{i_{2}=1}^{I_{2}} v_{i_{2}}^{2} x_{i_{2} j}^{2} \\
& \quad-\sum_{d_{1}=1}^{D_{1}} w_{d_{1}}^{1} z_{d_{1} j}^{1} \geq 0, j=1, \ldots, n, \\
& \sum_{r_{3}=1}^{R_{3}} u_{r_{3}}^{3} y_{r_{3} j}^{3}-\sum_{i_{3}=1}^{I_{3}} v_{i_{3}}^{3} x_{i_{3} j}^{3}-\sum_{d_{2}=1}^{D_{2}} w_{d_{2}}^{2} z_{d_{2} j}^{2} \geq 0, \\
& i_{i_{1}}, v_{i_{2}}^{2}, v_{i_{3}}^{3} \geq \varepsilon ; i_{1}=1, \ldots, I_{1} ; i_{2}=1, \ldots, I_{2} ; \\
& u_{r_{1}}^{1}, u_{r_{2}}^{2}, u_{r_{3}}^{3} \geq \varepsilon ; \quad r_{1}=1, \ldots, R_{1} ; r_{2}=1, \ldots, R_{2} ; \\
& r_{3}=1, \ldots, R_{3},
\end{aligned}
$$

In specifying the value of $\varphi_{o}^{1 F-\min }$ by Model 22, Model 13 is modified and converted to the model below.

\section{Model 23:}

$$
\begin{aligned}
\varphi_{o}^{12 F *}= & \min \left\{\varphi_{o}^{1 F} \cdot \varphi_{o}^{2 F} \mid \varphi_{j}^{L} \geq 1, \quad \varphi_{j}^{1 F} \geq 1,\right. \\
& \varphi_{j}^{2 F} \geq 1, \quad \varphi_{o}^{L}=\phi_{o}^{L *}, \quad \varphi_{o}^{1 F}=\frac{O_{o}^{2}}{I_{o}^{2}}, \\
& \left.\varphi_{o}^{1 F} \in\left[\phi_{o}^{1 F-\min }, M\right], \quad j=1, \ldots, n\right\} .
\end{aligned}
$$

It should be brought to attention that in Model 23, $\varphi_{o}^{1 F}$ is taken in the objective function as variable and like the optimistic approach, constraints which specify this variable, along with its interval of modification, are supplemented to the model. Model 23 is a fractional model and by using the Charnes-Cooper conversion (1962), it is converted into a linear model as given beneath:

\section{Model 24:}

$$
\varphi_{o}^{12 F *}=\min \varphi_{o}^{1 F} \cdot \sum_{r_{3}=1}^{R_{3}} u_{r_{3}}^{3} y_{r_{3} o}^{3}
$$

s.t.:

$$
\begin{gathered}
\sum_{i_{3}=1}^{I_{3}} v_{i_{3}}^{3} x_{i_{3} o}^{3}+\sum_{d_{2}=1}^{D_{2}} w_{d_{2}}^{2} z_{d_{2} o}^{2}=1 \\
\sum_{d_{1}=1}^{D_{1}} w_{d_{1}}^{1} z_{d_{1} j}^{1}-\sum_{r_{1}=1}^{R_{1}} u_{r_{1}}^{1} y_{r_{1} j}^{1}-\sum_{i_{1}=1}^{I_{1}} v_{i_{1}}^{1} x_{i_{1} j}^{1} \geq 0, \\
j=1, \ldots, n, \\
\sum_{d_{2}=1}^{D_{2}} w_{d_{2}}^{2} z_{d_{2} j}^{2}+\sum_{r_{2}=1}^{R_{2}} u_{r_{2}}^{2} y_{r_{2} j}^{2}-\sum_{i_{2}=1}^{I_{2}} v_{i_{2}}^{2} x_{i_{2} j}^{2} \\
-\sum_{d_{1}=1}^{D_{1}} w_{d_{1}}^{1} z_{d_{1} j}^{1} \geq 0, \quad j=1, \ldots, n,
\end{gathered}
$$

$\sum_{r_{3}=1}^{R_{3}} u_{r_{3}}^{3} y_{r_{3} j}^{3}-\sum_{i_{3}=1}^{I_{3}} v_{i_{3}}^{3} x_{i_{3} j}^{3}-\sum_{d_{2}=1}^{D_{2}} w_{d_{2}}^{2} z_{d_{2} j}^{2} \geq 0$

$j=1, \ldots, n$,

$$
\begin{aligned}
& \sum_{d_{1}=1}^{D_{1}} w_{d_{1}}^{1} z_{d_{1} o}^{1}-\sum_{r_{1}=1}^{R_{1}} u_{r_{1}}^{1} y_{r_{1} o}^{1}-\varphi_{o}^{L *} \sum_{i_{1}=1}^{I_{1}} v_{i_{1}}^{1} x_{i_{1} o}^{1}=0 \\
& \sum_{d_{2}=1}^{D_{2}} w_{d_{2}}^{2} z_{d_{2} o}^{2}+\sum_{r_{2}=1}^{R_{2}} u_{r_{2}}^{2} y_{r_{2} o}^{2}-\varphi_{o}^{1 F *} \\
& \quad\left(\sum_{i_{2}=1}^{I_{2}} v_{i_{2}}^{2} x_{i_{2} o}^{2}+\sum_{d_{1}=1}^{D_{1}} w_{d_{1}}^{1} z_{d_{1} o}^{1}\right)=0 \\
& \varphi_{o}^{1 F} \in\left[\varphi_{o}^{1 F-\min }, M\right] \\
& u_{r_{1}}^{1}, u_{r_{2}}^{2}, u_{r_{3}}^{3} \geq \varepsilon ; \quad r_{1}=1, \ldots, R_{1} ; \quad r_{2}=1, \ldots, R_{2} ; \\
& r_{3}=1, \ldots, R_{3}, \\
& v_{i_{1}}^{1}, v_{i_{2}}^{2}, v_{i_{3}}^{3} \geq \varepsilon ; \quad i_{1}=1, \ldots, I_{1} ; \quad i_{2}=1, \ldots, I_{2} ; \\
& i_{3}=1, \ldots, I_{3}, \\
& w_{d_{1}}^{1}, w_{d_{2}}^{2} \geq \varepsilon ; \quad d_{1}=1, \ldots, D_{1} ; \quad d_{2}=1, \ldots, D_{2} .
\end{aligned}
$$


In Model 24 and by employing Eq. (25), the value of $k_{1}$ is raised to its utmost level, in order to solve the model each time with the new $\varphi_{o}^{1 F}$. For the entire conditions of $k_{1}$, Model 24 is solved, and the responses of the model are assigned as $\varphi_{o}^{12 F}\left(k_{1}\right)$. By comparing all the values of $\varphi_{o}^{12 F}\left(k_{1}\right), \varphi_{o}^{12 F^{*}}$ is defined as the minimal efficiency of the follower stage from the pessimistic view. It should be noted that similar to the optimistic approach, the proposed approach has been tested under two conditions and each time a stage has been considered as a variable; with due attention to the fact that the efficiency of a stage is somewhat unique. Thus, the results of these two methods have been obtained with an extremely good approximation. In order to explicate this approach, one of these two conditions has been represented above.

\section{Case study description}

Throughout the past years, an increase in the importance of the production sector, and anxiety in regard to the development and efficiency growth of this segment has been directly correlated with that of the economic system. A rise in costs has led to forcing production units to improve their organizational performance. The optimal mode which would increase efficiency is to logically utilize, adopt and modify the available resources. This can only be achieved by ensuring correct managerial performance, including a rational evaluation of the returns attained $[54,55]$. The statistical population of this research includes the production, maintenance and distribution network of a factory (Nasiri Dairy factory), which is defined as an annual planning horizon in 24 periods. The factory is located in Nazar Abad Industrial Estate (in Iran), and its brand name is "Aramesh-e-Paitakht". This factory has a production area, a warehouse area and a delivery point, each of which is considered as a stage. The production area plays the role of the "leader" and the other two, the role of "followers". This factory was studied for of 24 time periods and as a dynamic network. In this network, a number of outputs during a time period of $t$ in the second stage are converted to a number of inputs in the second stage during a time period of $t+1$. Each time period is assumed to be a DMU. Hence, the inputs and outputs of each DMU are according to the following. The production costs of the three products produced are assigned as inputs of the first stage and are denoted as $\left(x_{1}^{1}, x_{2}^{1}, x_{3}^{1}\right)$. The transport costs for produce from the first to the second stage are described as undesirable output of the first stage, which is shown as $y_{1}^{1}$. The intermediary products between the first and second stages are the quantity of produce of each commodity, which are demonstrated as $\left(z_{1}^{1}, z_{2}^{1}, z_{3}^{1}\right)$. The additional outputs for the second stage are, respectively; the cost of reserving storage location $x_{1}^{2}$, cost of holding goods $x_{2}^{2}$ and the goods remaining in the warehouse from the previous period, which are illustrated as $\left(x_{3}^{2}, x_{4}^{2}, x_{5}^{2}\right)$. The outputs of the second stage are defined as the quantity of goods remaining in the warehouse for a subsequent period of time, and are represented by $\left(y_{1}^{2}, y_{2}^{2}, y_{3}^{2}\right)$. The intermediary products between the second and third stages are the quantities of delivery of each commodity, which are demonstrated by $\left(z_{1}^{4}, z_{1}^{5}, z_{1}^{6}\right)$. The additional input of the third stage are described as the transport costs of goods to the third stage and this is illustrated as $x_{1}^{3}$. Finally, the output of the third stage is the profit from the sale of goods, which is indicated by $y_{1}^{3}$. In continuation, he input values for the 24 time periods are illustrated in Table 2 and the mean values and outputs in Table 3.

In Table 2, the values of 0 , for each period, indicate that the goods have not remained in the warehouse since the previous phase (columns 7 to 9 ). Table 3 also shows values with 0 , which illustrate that the goods for the subsequent period have not remained in the warehouse (columns 9 to 11).

In continuation, the efficiency of the factory is secured by a black box approach and three leaderfollower scenarios. For this purpose and in the third scenario, $c_{1}=c_{2}=0.6$ and $c_{3}=c_{4}=1.05$ are considered as the goals of managers. The values of $\alpha_{1}$ have been obtained from Models 15 and 16 and show that the goals of the managers have been attained $\left(\alpha_{1}=0, i=1,2,3,4\right)$. In the second and third scenarios, on the basis of the opinions of managers, $\Delta \varepsilon=0.01$ and $M=5$ have been contemplated. Similarly, the value for $\varepsilon$ in all the models has been considered as 0.05 by the managers. The heuristic method has been executed as expressed in Section 3 for both second and third scenarios. The values achieved for $k_{1}$, together with maximal optimistic efficiency and minimal pessimistic efficiency of the first stage for the second and third scenarios, have been illustrated in Table 4.

In studying the values of $k_{1}$, it is known that, in this case-study, that the pessimistic efficiency values of all the followers in the second and third scenarios, in most cases, are optimized, when the values of $k_{1}$ are low (columns 4 and 8). This signifies that the optimal efficiency values of the second stage or the first follower are proximate to their minimum values (columns 5 and 8), whereas, in the case of the optimistic efficiency values of the second stage, are far from their maximum values, in most circumstances (columns 3 and 7). It should be noted that the closer the efficiency value is to 1 , from the optimistic standpoint, and the farther the efficiency value is from 1 , from the pessimistic standpoint, the DMU proves to have a better condition. Likewise, according to expectations, all efficiency values, both from the optimistic and pessimistic perspective, in the 
Table 2. The inputs of the factory for 24 periods in 2016

\begin{tabular}{|c|c|c|c|c|c|c|c|c|c|}
\hline \multirow[b]{2}{*}{ DMU } & \multicolumn{3}{|c|}{ Production cost } & \multirow{2}{*}{$\begin{array}{c}\text { Cost of } \\
\text { reserving } \\
\text { storage } \\
\text { location } \\
x_{1}^{2}\end{array}$} & \multirow{2}{*}{$\begin{array}{c}\begin{array}{c}\text { Cost of } \\
\text { holding } \\
\text { goods }\end{array} \\
x_{2}^{2} \\
\end{array}$} & \multicolumn{3}{|c|}{$\begin{array}{c}\text { Goods } \\
\text { remaining } \\
\text { from last } \\
\text { period }\end{array}$} & \multirow[t]{2}{*}{$\begin{array}{c}\text { Cost of } \\
\text { transport } \\
\text { goods to } \\
\text { delivery } \\
\text { points } \\
x_{1}^{3} \\
\end{array}$} \\
\hline & $x_{1}^{1}$ & $x_{2}^{1}$ & $x_{3}^{1}$ & & & $x_{3}^{2}$ & $x_{4}^{2}$ & $x_{5}^{2}$ & \\
\hline 1 & 29120000 & 36160000 & 51520000 & 1700000 & 1430000 & 0 & 0 & 0 & 3680000 \\
\hline 2 & 50960000 & 63280000 & 77280000 & 1700000 & 1430000 & 0 & 0 & 0 & 6235000 \\
\hline 3 & 80080000 & 99440000 & 128800000 & 1700000 & 1430000 & 0 & 0 & 0 & 9915000 \\
\hline 4 & 101920000 & 126560000 & 180320000 & 1700000 & 1430000 & 0 & 0 & 0 & 12880000 \\
\hline 5 & 43680000 & 54240000 & 77280000 & 1700000 & 1430000 & 0 & 0 & 0 & 5520000 \\
\hline 6 & 50960000 & 63280000 & 103040000 & 1700000 & 1430000 & 0 & 0 & 0 & 6645000 \\
\hline 7 & 94640000 & 126560000 & 154560000 & 1700000 & 1670000 & 0 & 0 & 0 & 11755000 \\
\hline 8 & 145600000 & 180800000 & 257600000 & 1700000 & 3620000 & 0 & 2 & 0 & 15435000 \\
\hline 9 & 145600000 & 180800000 & 257600000 & 1700000 & 3170000 & 6 & 8 & 4 & 19115000 \\
\hline 10 & 145600000 & 180800000 & 257600000 & 1700000 & 1730000 & 4 & 6 & 4 & 20555000 \\
\hline 11 & 145600000 & 180800000 & 257600000 & 1700000 & 1430000 & 0 & 0 & 2 & 19220000 \\
\hline 12 & 145600000 & 180800000 & 257600000 & 1700000 & 1430000 & 0 & 0 & 0 & 16815000 \\
\hline 13 & 87360000 & 99440000 & 128800000 & 1700000 & 1430000 & 0 & 0 & 0 & 10290000 \\
\hline 14 & 50960000 & 63280000 & 77280000 & 1700000 & 1430000 & 0 & 0 & 0 & 6235000 \\
\hline 15 & 50960000 & 63280000 & 103040000 & 1700000 & 1430000 & 0 & 0 & 0 & 6645000 \\
\hline 16 & 43680000 & 54240000 & 77280000 & 1700000 & 1430000 & 0 & 0 & 0 & 5520000 \\
\hline 17 & 80080000 & 99440000 & 128800000 & 1700000 & 1430000 & 0 & 0 & 0 & 9915000 \\
\hline 18 & 94640000 & 117520000 & 154560000 & 1700000 & 1430000 & 0 & 0 & 0 & 11755000 \\
\hline 19 & 72800000 & 90400000 & 128800000 & 1700000 & 1430000 & 0 & 0 & 0 & 9200000 \\
\hline 20 & 87360000 & 108480000 & 154560000 & 1700000 & 1430000 & 0 & 0 & 0 & 11040000 \\
\hline 21 & 87360000 & 108480000 & 128800000 & 1700000 & 1430000 & 0 & 0 & 0 & 10630000 \\
\hline 22 & 109200000 & 135600000 & 180320000 & 1700000 & 3830000 & 0 & 0 & 0 & 9915000 \\
\hline 23 & 145600000 & 180800000 & 257600000 & 1700000 & 1430000 & 8 & 8 & 4 & 22080000 \\
\hline 24 & 145600000 & 180800000 & 257600000 & 1700000 & 1430000 & 0 & 0 & 0 & 18400000 \\
\hline
\end{tabular}

third leader-follower scenario are higher than the level of goals 0.6,1.05. Tables 5 and 6 render the optimistic and pessimistic efficiency values of the stages for the three "leader-follower" scenarios, respectively.

In Table 5, columns 2, 5 and 8 demonstrate the efficiency values of the leader stage using the optimistic approach. In this case study, the efficiency values of the leader stage for all DMUs, in each of the three leader-follower scenarios, equate to 1. In general, and with due attention to the two constraints supplemented into the third scenario, with respect to the optimistic efficiency values of the leader stage, equation $\theta_{\mathrm{o}}^{\text {Lscenario3 }} \leq \theta_{\mathrm{o}}^{\text {Lscenario1 }}=\theta_{\mathrm{o}}^{\text {Lscenario2 }}$ is expected. In comparing the efficiency values of the followers in this case study, it is observed that the optimistic efficiency values gained for the first scenario (including all DMUs) are more than the second and third scenarios $\left(\theta_{\mathrm{o}}^{1 \mathrm{Fscenario} 2}, \theta_{\mathrm{o}}^{1 \text { Fscenario3 }} \leq\right.$ $\theta_{\mathrm{o}}^{1 \text { Fscenario } 1}$ and $\left.\theta_{\mathrm{o}}^{2 \text { Fscenario } 2}, \theta_{\mathrm{o}}^{2 \text { Fscenario } 3} \leq \theta_{\mathrm{o}}^{2 \text { Fscenario } 1}\right)$. Likewise, as expected, results show that the optimistic efficiency values of the followers for all DMUs in the second scenario are more than the third scenario $\left(\theta_{\mathrm{o}}^{12 \text { Fscenario3 }} \leq \theta_{\mathrm{o}}^{12 \text { Fscenario2 }}\right)$. This is because of the two additional constraints of the third scenario. Similarly, results also illustrate that the average optimistic efficiency values of the leader, first follower and second follower in the three leader-follower scenarios are of the following values, respectively $(1,0.98,0.91)$, $(1,0.82,0.63)$ and $(1,0.80,0.64)$. As a result, in all three scenarios, the average optimistic efficiency values of the leader are higher than those of the followers. Likewise, the average optimistic efficiency values of the first leader, which were obtained, are more than the second follower. These results are 
Table 3. The outputs and the intermediate measures of the factory for 24 periods in 2016 .

\begin{tabular}{|c|c|c|c|c|c|c|c|c|c|c|c|}
\hline \multirow[b]{2}{*}{ DMU } & \multicolumn{3}{|c|}{$\begin{array}{l}\text { Quantity of } \\
\text { goods } \\
\text { produced }\end{array}$} & \multicolumn{3}{|c|}{$\begin{array}{l}\text { Quantity of } \\
\text { goods } \\
\text { delivered }\end{array}$} & \multirow{2}{*}{$\begin{array}{c}\text { Cost of } \\
\text { transport } \\
\text { goods to } \\
\text { warehouse } \\
y_{1}^{1} \\
\end{array}$} & \multicolumn{3}{|c|}{$\begin{array}{l}\text { Goods } \\
\text { remaining for } \\
\text { next period }\end{array}$} & \multirow{2}{*}{$\begin{array}{c}\text { Profit } \\
y_{1}^{3} \\
\end{array}$} \\
\hline & $z_{1}^{1}$ & $z_{2}^{1}$ & $z_{3}^{1}$ & $z_{1}^{2}$ & $z_{2}^{2}$ & $z_{3}^{2}$ & & $y_{1}^{2}$ & $y_{2}^{2}$ & $y_{3}^{2}$ & \\
\hline 1 & 8 & 8 & 4 & 8 & 8 & 4 & 1960000 & 0 & 0 & 0 & 31800000 \\
\hline 2 & 14 & 14 & 6 & 14 & 14 & 6 & 3310000 & 0 & 0 & 0 & 51110000 \\
\hline 3 & 22 & 22 & 10 & 22 & 22 & 10 & 5270000 & 0 & 0 & 0 & 82910000 \\
\hline 4 & 28 & 28 & 14 & 28 & 28 & 14 & 6860000 & 0 & 0 & 0 & 111300000 \\
\hline 5 & 12 & 12 & 6 & 12 & 12 & 6 & 2940000 & 0 & 0 & 0 & 47700000 \\
\hline 6 & 14 & 14 & 8 & 14 & 14 & 8 & 3550000 & 0 & 0 & 0 & 60190000 \\
\hline 7 & 26 & 28 & 12 & 26 & 26 & 12 & 6460000 & 0 & 2 & 0 & 98810000 \\
\hline 8 & 40 & 40 & 20 & 34 & 34 & 16 & 9800000 & 6 & 8 & 4 & 130610000 \\
\hline 9 & 40 & 40 & 20 & 42 & 42 & 20 & 9800000 & 4 & 6 & 4 & 162410000 \\
\hline 10 & 40 & 40 & 20 & 44 & 46 & 22 & 9800000 & 0 & 0 & 2 & 177380000 \\
\hline 11 & 40 & 40 & 20 & 40 & 40 & 22 & 9800000 & 0 & 0 & 0 & 166880000 \\
\hline 12 & 40 & 40 & 20 & 34 & 40 & 20 & 9800000 & 0 & 0 & 0 & 153510000 \\
\hline 13 & 24 & 22 & 10 & 24 & 22 & 10 & 5430000 & 0 & 0 & 0 & 83640000 \\
\hline 14 & 14 & 14 & 6 & 14 & 14 & 6 & 3310000 & 0 & 0 & 0 & 51110000 \\
\hline 15 & 14 & 14 & 8 & 14 & 14 & 8 & 3550000 & 0 & 0 & 0 & 60190000 \\
\hline 16 & 12 & 12 & 6 & 12 & 12 & 6 & 2940000 & 0 & 0 & 0 & 47700000 \\
\hline 17 & 22 & 22 & 10 & 22 & 22 & 10 & 5270000 & 0 & 0 & 0 & 82910000 \\
\hline 18 & 26 & 26 & 12 & 26 & 26 & 12 & 6250000 & 0 & 0 & 0 & 98810000 \\
\hline 19 & 20 & 20 & 10 & 20 & 20 & 10 & 4900000 & 0 & 0 & 0 & 79500000 \\
\hline 20 & 24 & 24 & 12 & 24 & 24 & 12 & 5880000 & 0 & 0 & 0 & 95400000 \\
\hline 21 & 24 & 24 & 10 & 24 & 24 & 10 & 5640000 & 0 & 0 & 0 & 86320000 \\
\hline 22 & 30 & 30 & 14 & 22 & 22 & 10 & 7230000 & 8 & 8 & 4 & 82910000 \\
\hline 23 & 40 & 40 & 20 & 48 & 48 & 24 & 9800000 & 0 & 0 & 0 & 190800000 \\
\hline 24 & 40 & 40 & 20 & 40 & 40 & 20 & 9800000 & 0 & 0 & 0 & 159000000 \\
\hline
\end{tabular}

acceptable or appropriate due to the priority status of the stages.

In Table 6, columns (2, 5, and 8) demonstrate that the pessimistic efficiency values of the leader stage in each of the three scenarios are equal to 1 . The objective function of the pessimistic efficiency is minimizing. In general, it is expected that, by being attentive, in respect to the two constraints added to the third scenario, equation $\varphi_{o}^{\text {Lscenario1 }}=\varphi_{o}^{\text {Lscenario2 }} \leq \varphi_{o}^{\text {Lscenario3 }}$ will be confirmed. In comparing the efficiency values of the first follower in this case study, it is observed that the pessimistic efficiency values obtained from the first scenario for all DMUs are less than the second and third scenarios $\left(\varphi_{0}^{1 \text { Fscenario } 1} \leq \varphi_{0}^{1 \text { Fscenario2 }}\right.$ and $\left.\varphi_{o}^{1 \text { Fscenario3 }}\right)$. The pessimistic efficiency values attained from the second scenario for the second follower are less than the first and third scenarios $\left(\varphi_{o}^{2 \text { Fscenario2 }} \leq\right.$

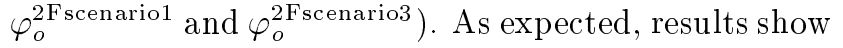
that the pessimistic efficiency values of the followers for all DMUs in the second scenario are less than the third scenario. This is due to the two additional constraints of the third scenario $\left(\varphi_{o}^{12 \text { Fscenario } 2} \leq \varphi_{o}^{12 \text { Fscenario3 }}\right)$. Results demonstrate that the average pessimistic efficiency values of the leader and the first and second followers in the three leader-follower scenarios are $(1,1.05,1.04),(1,1.08,1.007)$ and $(1,1.10,1.05)$, respectively. This results in the fact that in each of the three scenarios, the pessimistic average efficiency values of the leader are less than the followers and, similarly, the average pessimistic efficiency values attained for the first follower are more than that of the second follower. As expected, in the third scenario, the pessimistic efficiency values in al followers are higher than the level of goals by (1.05). For each view and scenario, the optimistic, pessimistic and dual-frontier overall efficiency values have been described, the results of which have been rendered in Table 7 .

In comparing the optimistic overall efficiency 
Table 4. Results of the maximum and minimum efficiencies of the first stage and $k$ values.

\begin{tabular}{|c|c|c|c|c|c|c|c|c|}
\hline \multirow[b]{3}{*}{ DMU } & \multicolumn{4}{|c|}{ Second leader-follower scenario } & \multicolumn{4}{|c|}{ Third leader-follower scenario } \\
\hline & \multicolumn{2}{|c|}{ Optimistic view } & \multicolumn{2}{|c|}{ Pessimistic view } & \multicolumn{2}{|c|}{ Optimistic view } & \multicolumn{2}{|c|}{ Pessimistic view } \\
\hline & $k_{1}$ & $\theta_{o}^{1 F-\max }$ & $k_{1}$ & $\varphi_{o}^{1 F-\min }$ & $k_{1}$ & $\theta_{o}^{1 F-\max }$ & $k_{1}$ & $\varphi_{0}^{1 F-\min }$ \\
\hline 1 & 26 & 0.94507 & 0 & 1 & 26 & 0.94507 & 0 & 1.05 \\
\hline 2 & 17 & 0.96910 & 0 & 1 & 17 & 0.9691 & 0 & 1.05 \\
\hline 3 & 22 & 0.98283 & 1 & 1.06867 & 19 & 0.98283 & 1 & 1.06867 \\
\hline 4 & 13 & 0.97940 & 0 & 1.17167 & 17 & 0.9794 & 5 & 1.17167 \\
\hline 5 & 20 & 0.95193 & 1 & 1.03433 & 20 & 0.95193 & 0 & 1.05 \\
\hline 6 & 18 & 1 & 2 & 1 & 18 & 1 & 0 & 1.05 \\
\hline 7 & 16 & 1 & 2 & 1.05053 & 17 & 1 & 2 & 1.05053 \\
\hline 8 & 0 & 1 & 2 & 1.08231 & 0 & 1 & 0 & 1.08231 \\
\hline 9 & 0 & 1 & 0 & 1 & 0 & 1 & 0 & 1.05 \\
\hline 10 & 9 & 1 & 0 & 1 & 9 & 1 & 0 & 1.05 \\
\hline 11 & 14 & 1 & 10 & 1.08356 & 39 & 1 & 7 & 1.08356 \\
\hline 12 & 6 & 0.99375 & 22 & 1.09597 & 26 & 0.99375 & 20 & 1.09597 \\
\hline 13 & 20 & 1 & 2 & 1 & 17 & 1 & 0 & 1.05 \\
\hline 14 & 17 & 0.9691 & 0 & 1 & 17 & 0.9691 & 0 & 1.05 \\
\hline 15 & 18 & 1 & 2 & 1 & 18 & 1 & 0 & 1.05 \\
\hline 16 & 20 & 0.95193 & 1 & 1.03433 & 20 & 0.95193 & 0 & 1.05 \\
\hline 17 & 22 & 0.98283 & 1 & 1.06867 & 19 & 0.98283 & 1 & 1.06867 \\
\hline 18 & 16 & 0.98970 & 3 & 1.103 & 17 & 0.9897 & 2 & 1.103 \\
\hline 19 & 23 & 0.96567 & 0 & 1.103 & 20 & 0.96567 & 2 & 1.103 \\
\hline 20 & 18 & 0.97253 & 0 & 1.13734 & 15 & 0.97253 & 3 & 1.13734 \\
\hline 21 & 20 & 1 & 2 & 1.02199 & 19 & 1 & 0 & 1.05 \\
\hline 22 & 0 & 1 & 0 & 1 & 0 & 1 & 0 & 1.05 \\
\hline 23 & 38 & 1 & 0 & 1 & 40 & 1 & 0 & 1.05 \\
\hline 24 & 6 & 1 & 3 & 1.27467 & 33 & 1 & 13 & 1.27467 \\
\hline
\end{tabular}

of the black box view and the three leader-follower scenarios, by utilizing the Table 7 , it can be observed that the black box view had the maximal efficiency for all DMUs. This arises because the black box view fails to consider the intermediary variables. The first scenario of the leader-follower aspects shows optimistic overall efficiency with a slight difference and diminution than the black box view, and is in second place. The optimistic overall efficiency computed by the second and third leader-follower scenarios is in the third and fourth position, respectively. But, in most cases, has an outstanding difference from the viewpoint of the optimistic overall efficiency performance with the two scenarios, i.e. the first and second scenarios. The reason as to why the third leader-follower scenario is lower than the second scenario is because the goals of the managers are imposed in the models of the third scenario. Hence, by comparing columns 2, 5, 8, and 11 in Table 7, it can be noted that the optimistic overall efficiency performance computed in this case study for 4 viewpoints is accordingly:

$$
\begin{aligned}
& \theta_{o}^{\text {overall-scenario3 }} \leq \theta_{o}^{\text {overall-scenario2 }} \\
& \quad \leq \theta_{o}^{\text {overall-scenario1 }} \leq \theta_{o}^{\text {overall-blackbox }} .
\end{aligned}
$$

In Table 7, by comparing columns 3, 6, 9, and 12 , which demonstrate the pessimistic overall efficiency, it is known that in this case study, the results of the black box view has a minimal distance from the inefficient frontier in comparison to the three other scenarios, which, contrary to the optimistic view, causes it to earn the poorest or worst position. Likewise, in comparing the pessimistic overall efficiency of the leader-follower in the second and third scenarios, it is understood that the pessimistic overall efficiency which has been obtained from the third scenario, is more than that of the 
Table 5. The efficiency values of stages in the three leader-follower scenarios from the optimistic approach.

\begin{tabular}{|c|c|c|c|c|c|c|c|c|c|}
\hline \multirow[b]{2}{*}{ DMU } & \multicolumn{3}{|c|}{$\begin{array}{c}\text { First leader-follower } \\
\text { scenario }\end{array}$} & \multicolumn{3}{|c|}{$\begin{array}{c}\text { Second leader-follower } \\
\text { scenario }\end{array}$} & \multicolumn{3}{|c|}{$\begin{array}{c}\text { Third leader-follower } \\
\text { scenario }\end{array}$} \\
\hline & $\theta_{o}^{L}$ & $\theta_{o}^{1 F}$ & $\theta_{o}^{2 F}$ & $\theta_{o}^{L}$ & $\theta_{o}^{1 F}$ & $\theta_{o}^{2 F}$ & $\theta_{o}^{L}$ & $\theta_{o}^{1 F}$ & $\theta_{o}^{2 F}$ \\
\hline 1 & 1 & 0.94507 & 0.91195 & 1 & 0.68507 & 0.72563 & 1 & 0.68507 & 0.72563 \\
\hline 2 & 1 & 0.9691 & 0.86468 & 1 & 0.7991 & 0.61713 & 1 & 0.7991 & 0.61713 \\
\hline 3 & 1 & 0.98283 & 0.88133 & 1 & 0.79283 & 0.63265 & 1 & 0.79283 & 0.63265 \\
\hline 4 & 1 & 0.9794 & 0.91057 & 1 & 0.8794 & 0.56568 & 1 & 0.8094 & 0.60449 \\
\hline 5 & 1 & 0.95193 & 0.91168 & 1 & 0.75193 & 0.67144 & 1 & 0.75193 & 0.67144 \\
\hline 6 & 1 & 1 & 0.97593 & 1 & 0.82 & 0.68081 & 1 & 0.82 & 0.68081 \\
\hline 7 & 1 & 1 & 0.90633 & 1 & 0.87 & 0.58235 & 1 & 0.83 & 0.60559 \\
\hline 8 & 1 & 1 & 0.92294 & 1 & 1 & 0.72931 & 1 & 1 & 0.72931 \\
\hline 9 & 1 & 1 & 0.92576 & 1 & 1 & 0.68483 & 1 & 1 & 0.68483 \\
\hline 10 & 1 & 1 & 0.92942 & 1 & 0.91 & 0.67609 & 1 & 0.91 & 0.67609 \\
\hline 11 & 1 & 1 & 0.93714 & 1 & 0.76 & 0.50927 & 1 & 0.61 & 0.60542 \\
\hline 12 & 1 & 0.99375 & 0.96025 & 1 & 0.82375 & 0.54205 & 1 & 0.73375 & 0.60002 \\
\hline 13 & 1 & 1 & 0.87972 & 1 & 0.84 & 0.5945 & 1 & 0.83 & 0.60109 \\
\hline 14 & 1 & 0.9691 & 0.86468 & 1 & 0.7991 & 0.61713 & 1 & 0.7991 & 0.61713 \\
\hline 15 & 1 & 1 & 0.97593 & 1 & 0.82 & 0.68081 & 1 & 0.82 & 0.68081 \\
\hline 16 & 1 & 0.95193 & 0.91168 & 1 & 0.75193 & 0.67144 & 1 & 0.75193 & 0.67144 \\
\hline 17 & 1 & 0.98283 & 0.88133 & 1 & 0.79283 & 0.63265 & 1 & 0.79283 & 0.63265 \\
\hline 18 & 1 & 0.9897 & 0.8856 & 1 & 0.8497 & 0.58492 & 1 & 0.8197 & 0.60045 \\
\hline 19 & 1 & 0.96567 & 0.91112 & 1 & 0.76567 & 0.66846 & 1 & 0.76567 & 0.66846 \\
\hline 20 & 1 & 0.97253 & 0.91085 & 1 & 0.82253 & 0.62146 & 1 & 0.82253 & 0.62146 \\
\hline 21 & 1 & 1 & 0.87839 & 1 & 0.83 & 0.59195 & 1 & 0.81 & 0.60153 \\
\hline 22 & 1 & 1 & 0.91345 & 1 & 1 & 0.79076 & 1 & 1 & 0.79076 \\
\hline 23 & 1 & 1 & 0.91335 & 1 & 0.57 & 0.63178 & 1 & 0.6 & 0.6 \\
\hline 24 & 1 & 1 & 0.93563 & 1 & 0.83 & 0.51003 & 1 & 0.67 & 0.60471 \\
\hline
\end{tabular}

second one. This is due to the goals of managers; which, in contrast to the optimistic approach of the third scenario, holds a better condition in comparison to the second scenario. By comparing these two scenarios with the first leader-follower scenario, a specific result cannot be outlined. Given the differences in the results of the optimistic and pessimistic approaches, for a final summarization of these 4 viewpoints, see columns 4 , 7,10 , and 13 of Table 7 , which illustrates efficiency by taking the double-frontier into consideration. It is noted that in this case study, the black box view and the leader-follower of the first scenario provided close results for the network shown in Figure 1, but with this variance; that the first leader-follower scenario takes the intermediate variables under consideration. In the black box view, however, in order to alleviate matters, it is neglected. Similarly, by considering the goals of managers, the results of the second and third leader- follower scenarios are in approximation to each other, whilst there is a significant difference with the two other scenarios.

Given, that this paper proposes a double-frontier approach in order to measure overall efficiency, the best and poorest DMUs in accordance with the black box scenario are units 24 and 1 with overall efficiencies of 1.12901 and 0.97214 , respectively. On the basis of the first leader-follower scenario, unit 12, with a total efficiency of 1.18253 , is the best; whereas units 2 and 14, displaying a common and overall efficiency of 0.91598 , are the worst units. It should be noted that, in some cases, for example, in $D M U_{2}$ and $D M U_{14}$, the rank of the DMUs are equal. This is due to the fact that the demand, the amount of production of each type of goods, the amount of delivery and maintenance of each type of goods and other items during periods 2 and 14 were absolutely equivalent. In accordance with the 
Table 6. The efficiency values of stages in the three leader-follower scenarios from the pessimistic approach.

\begin{tabular}{|c|c|c|c|c|c|c|c|c|c|}
\hline \multirow[b]{2}{*}{ DMU } & \multicolumn{3}{|c|}{$\begin{array}{c}\text { First leader-follower } \\
\text { scenario }\end{array}$} & \multicolumn{3}{|c|}{$\begin{array}{c}\text { Second leader-follower } \\
\text { scenario }\end{array}$} & \multicolumn{3}{|c|}{$\begin{array}{c}\text { Third leader-follower } \\
\text { scenario }\end{array}$} \\
\hline & $\varphi_{o}^{L}$ & $\varphi_{0}^{1 F}$ & $\varphi_{o}^{2 F}$ & $\varphi_{0}^{L}$ & $\varphi_{0}^{1 F}$ & $\varphi_{o}^{2 F}$ & $\varphi_{o}^{L}$ & $\varphi_{0}^{1 F}$ & $\varphi_{o}^{2 F}$ \\
\hline 1 & 1 & 1 & 1.00781 & 1 & 1 & 1.00781 & 1 & 1.05 & 1.05 \\
\hline 2 & 1 & 1 & 1.00128 & 1 & 1 & 1.00128 & 1 & 1.05 & 1.05 \\
\hline 3 & 1 & 1.06867 & 1.0182 & 1 & 1.07867 & 1.00708 & 1 & 1.07867 & 1.05 \\
\hline 4 & 1 & 1.17167 & 1.00785 & 1 & 1.17167 & 1.00785 & 1 & 1.22167 & 1.05 \\
\hline 5 & 1 & 1.03433 & 1.00781 & 1 & 1.04433 & 1.00781 & 1 & 1.05 & 1.05 \\
\hline 6 & 1 & 1 & 1.04651 & 1 & 1.02 & 1.00966 & 1 & 1.05 & 1.05 \\
\hline 7 & 1 & 1.05182 & 1.04226 & 1 & 1.07053 & 1.01118 & 1 & 1.07053 & 1.05 \\
\hline 8 & 1 & 1.08231 & 1.04466 & 1 & 1.10231 & 1.00763 & 1 & 1.08231 & 1.05 \\
\hline 9 & 1 & 1 & 1.00626 & 1 & 1 & 1.00626 & 1 & 1.05 & 1.05 \\
\hline 10 & 1 & 1 & 1.00775 & 1 & 1 & 1.00775 & 1 & 1.05 & 1.05 \\
\hline 11 & 1 & 1.08356 & 1.20643 & 1 & 1.18356 & 1.00223 & 1 & 1.15356 & 1.0558 \\
\hline 12 & 1 & 1.09597 & 1.33712 & 1 & 1.31597 & 1.02494 & 1 & 1.29597 & 1.05 \\
\hline 13 & 1 & 1 & 1.03943 & 1 & 1.02 & 1 & 1 & 1.05 & 1.05 \\
\hline 14 & 1 & 1 & 1.00128 & 1 & 1 & 1.00128 & 1 & 1.05 & 1.05 \\
\hline 15 & 1 & 1 & 1.04651 & 1 & 1.02 & 1.00966 & 1 & 1.05 & 1.05 \\
\hline 16 & 1 & 1.03433 & 1.00781 & 1 & 1.04433 & 1.00781 & 1 & 1.05 & 1.05 \\
\hline 17 & 1 & 1.06867 & 1.0182 & 1 & 1.07867 & 1.00708 & 1 & 1.07867 & 1.05 \\
\hline 18 & 1 & 1.103 & 1.05095 & 1 & 1.133 & 1.00734 & 1 & 1.123 & 1.05 \\
\hline 19 & 1 & 1.103 & 1.00783 & 1 & 1.103 & 1.00783 & 1 & 1.123 & 1.05 \\
\hline 20 & 1 & 1.13734 & 1.00784 & 1 & 1.13734 & 1.00784 & 1 & 1.16734 & 1.05 \\
\hline 21 & 1 & 1.02216 & 1.15101 & 1 & 1.04199 & 1.00791 & 1 & 1.05 & 1.05 \\
\hline 22 & 1 & 1 & 1.00397 & 1 & 1 & 1.00397 & 1 & 1.05 & 1.05 \\
\hline 23 & 1 & 1 & 1.00789 & 1 & 1 & 1.00789 & 1 & 1.05 & 1.05 \\
\hline 24 & 1 & 1.27467 & 1 & 1 & 1.30467 & 1.00787 & 1 & 1.40467 & 1.05 \\
\hline
\end{tabular}

second scenario, the leader-follower aspect of units 8 and 23 , with overall efficiencies of 0.90003 and 0.60246 , are the optimum and the poorest units, respectively. Ultimately, based on the leader-follower facet of the third scenario, unit 22 , with a total efficiency of 0.9337 , is the best, and unit 23, with an overall efficiency of 0.63 , is the worst unit.

\section{Conclusion}

In this paper a three-stage network has been considered with additional inputs and outputs that are desirable and undesirable. The efficiency of this network has been computed using the black box approach and three leader-follower scenarios. In the black box view, the entire system is considered as a leader without any followers. In the first leader-follower scenario, the first, second and third stages were contemplated as "leader", "first follower" and "second follower", respectively. In this scenario, two roles are considered for the second stage:

1. Follower of the first stage;

2. Leader of the third stage.

In the second and third scenarios, assigned as leaderfollower, the first stage is considered a leader, and the other two stages together as a follower. In this paper, a manufacturing factory with a production and warehouse area, including a delivery point for goods, was taken under consideration. The total costs, comprising production costs, storage costs, reservation of warehouse (space) costs, and transportation costs, from both the production area to the warehouse and from the warehouse to the delivery point of goods, including the profits from the sale of commodities, 
Table 7. A comparison of the black box view and the three leader-follower scenarios.

\begin{tabular}{|c|c|c|c|c|c|c|c|c|c|c|c|c|}
\hline \multirow[b]{2}{*}{ DMU } & \multicolumn{3}{|c|}{$\begin{array}{c}\text { Black } \\
\text { box view }\end{array}$} & \multicolumn{3}{|c|}{$\begin{array}{c}\text { First leader-follower } \\
\text { scenario }\end{array}$} & \multicolumn{3}{|c|}{$\begin{array}{c}\text { Second leader-follower } \\
\text { scenario }\end{array}$} & \multicolumn{3}{|c|}{$\begin{array}{l}\text { Third leader-follower } \\
\text { scenario }\end{array}$} \\
\hline & $\theta_{o}^{\text {overall }}$ & $\varphi_{o}^{\text {overall }}$ & $\phi_{o}^{o v e r a l l}$ & $\theta_{o}^{\text {overall }}$ & $\varphi_{o}^{\text {overall }}$ & & & $\varphi_{o}^{\text {overall }}$ & & & & \\
\hline 1 & 0.94507 & 1 & 0.97214 & 0.86185 & 1.00781 & 0.93198 & 0.49711 & 1.00781 & 0.7078 & .00 .49711 & 1.1025 & 0.74031 \\
\hline 2 & & 1 & & & & & & & & & & 0.73735 \\
\hline 3 & 8283 & 1.068 & & 19 & & & & & & 09 & & 5372 \\
\hline 4 & & $1.1^{\prime}$ & & & & & & & & & & 9222 \\
\hline 5 & 5193 & 03433 & & 86785 & & & & & & & & 1607 \\
\hline 6 & 1 & 1.01845 & 0918 & 97593 & & & 5827 & & & & & 153 \\
\hline 7 & 1 & 1 & 1 & 0.90633 & 9626 & 0.99678 & 50665 & 825 & 4057 & .50264 & 406 & 0.75166 \\
\hline 8 & 1 & 1 & 1 & 92294 & 1. & 1.02152 & 72931 & & 90003 & 72931 & 43 & 0.91038 \\
\hline 9 & 1 & 1 & te & & & & & & & & & \\
\hline 10 & 1 & 1 & 1 & & & & & & & & & 0.02509 \\
\hline 11 & 1 & 1 & 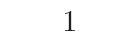 & 93714 & 1.30723 & 0682 & 8705 & & 7758 & 0.36931 & 794 & 0.67066 \\
\hline 12 & 1 & 11741 & -7 & .95424 & 1.46544 & 8253 & 14652 & 1.3488 & 0.77605 & 0.44027 & 6077 & 0.77401 \\
\hline 13 & & 1 & & & & & & & & & & 165 \\
\hline 14 & - & 1 & & & & & & & & & & 35 \\
\hline 15 & 1 & 1.01 & & 593 & & & 827 & & & & & 8453 \\
\hline 16 & 5193 & 1.03433 & 1227 & 0.86785 & 1.0424 & 6113 & 488 & 1. & 895 & 0.50488 & 25 & 0.74607 \\
\hline 17 & & 1.06867 & & & & & & & & & & 0.75372 \\
\hline 18 & & & & & & & & & & & & 0.76181 \\
\hline 19 & 0.96567 & 1.103 & & & & & & & & & 15 & 0.77686 \\
\hline 20 & 0.97253 & 1.13734 & 1.05171 & 0.8 & & 1. & 17 & & 6 & 17 & 71 & 0.79154 \\
\hline 21 & 1 & 1 & 1 & 0.87839 & 1.17651 & 1.01658 & 0.49132 & 24 & 0.71833 & 0.48724 & 025 & 0.73292 \\
\hline 22 & 1 & 1 & 1 & 0.91345 & 1.00397 & 0.95764 & 0.79076 & 1.00397 & 0.89101 & 0.79076 & 1.1025 & 0.9337 \\
\hline 23 & 1 & 1 & 1 & 0.91335 & 1.00789 & 0.95945 & 0.36012 & 1.00789 & 0.60246 & 0.36 & 1.1025 & 0.63 \\
\hline 24 & 1 & 1.27467 & 1.12901 & 0.93563 & 1.27467 & 1.09207 & 0.42333 & 1.31494 & 0.74609 & 0.40516 & 1.4749 & 0.77302 \\
\hline
\end{tabular}

have been simulated. This factory is considered a dynamic network consisting of 24 intervals. In order to achieve better accuracy, the optimistic and pessimistic approaches are utilized to evaluate efficiency. In this paper, a heuristic method is used to solve complex models in the leader-follower aspect of the second and third scenarios.

In this paper, an effort has been made to assist managers in network analysis by utilizing diverse noncooperative approaches; and also insert the goals of managers in the models. It was for this purpose that the Goal Programming (GP) concept was pursued in the third leader-follower scenario; and a leaderfollower collaboration was defined based on the goals of managers, which distinguishes this scenario from that of the second scenario. The black box view was observed and the first leader-follower scenario showed close results for the network, with the disparity that, in this scenario, the intermediary variables were taken into consideration, whereas, in the black box view, this was not so. Likewise, due to the goals of managers which have been contemplated in the second scenario, the results of the second and third leaderfollower scenarios are in approximation with each other, but have significant differences with the other two scenarios. It is quite evident that if the values of the goals of managers $\left(a_{i}, i=1,2,3,4\right)$ are modified, the results of the third leader-follower scenario can exhibit even more exceptional differences with respect to the second leader-follower scenario. The conditions necessary to ascertain the goals of managers are when the values of $\left(c_{i}, i=1,2,3,4\right)$ are equivalent to 0 .

The proposed heuristic approach in this paper was performed for three stages and due to the presence of additional inputs and outputs, the stages increased, thereby, making the model more complicated. As a result, the solving period is extremely elevated. In order to decrease this period, step size $(\Delta \varepsilon)$ can be increased. Hence, the value of step size $(\Delta \varepsilon)$, which determines the accuracy and the time for resolving the problem, can be considered by managers. The results of this research are put at the disposal of the 
managers, so that they procure the best decision for the abovementioned factory. Modeling with inaccurate and random data is suggested for research in the future.

\section{References}

1. Hwang, S.N., Chen, C., Chen, Y., Lee, H.S., and Shen, P.D. "Sustainable design performance evaluation with applications in the automobile industry: Focusing on inefficiency by undesirable factors", Omega, 41(3), pp. 553-558 (2013).

2. Kritikos, M.N. "A full ranking methodology in data envelopment analysis based on a set of dummy decision making units", Expert Systems with Applications, 77, pp. 211-225 (2017).

3. Charnes, A., Cooper, W.W., and Rhodes, E. "Measuring the efficiency of decision making units", European Journal of Operational Research, 2(6) pp. 429-444 (1978).

4. Farrell, M.J. "The measurement of productive efficiency", Journal of the Royal Statistical Society: Series A (General), 120(3), pp. 253-281 (1957).

5. Banker, R.D., Charnes, A., and Cooper, W.W. "Some models for estimating technical and scale inefficiencies in data envelopment analysis", Management Science, 30(9), pp. 1078-1092 (1984).

6. Lee, T., Zhang, Y., and Jeong, B.H. "A multi-period output DEA model with consistent time lag effects", Computers \& Industrial Engineering, 93, pp. 267-274 (2016).

7. Färe, R., Grosskopf, S., and Pasurka Jr, C.A. "Accounting for air pollution emissions in measures of state manufacturing productivity growth", Journal of Regional Science, 41(3), pp. 381-409 (2001).

8. Kou, M., Chen, K., Wang, S., and Shao, Y. "Measuring efficiencies of multi-period and multi-division systems associated with DEA: An application to OECD countries national innovation systems", Expert Systems with Applications, 46, pp. 494-510 (2016).

9. Wanke, P. and Barros, C. "Two-stage DEA: An application to major Brazilian banks", Expert Systems with Applications, 41(5), pp. 2337-2344 (2014).

10. Liang, L., Cook, W.D., and Zhu, J. "DEA models for two-stage processes: Game approach and efficiency decomposition", Naval Research Logistics (NRL), 55(7), pp. 643-653 (2008).

11. Li, Y., Chen, Y., Liang, L., and Xie, J. "DEA models for extended two-stage network structures", Omega, 40(5), pp. 611-618 (2012).

12. An, Q., Yang, M., Chu, J., Wu, J., and Zhu, Q. "Efficiency evaluation of an interactive system by data envelopment analysis approach", Computers \& Industrial Engineering, 103, pp. 17-25 (2017).

13. Wu, J., Zhu, Q., Ji, X., Chu, j., and Liang, L. "Two-stage network processes with shared resources and resources recovered from undesirable outputs", European Journal of Operational Research, 251(1), pp. 182-197 (2016).
14. Zhou, X., Luo, R., Tu, Y., Lev, B., and Pedrycz, W. "Data envelopment analysis for bi-level systems with multiple followers", Omega, 77, pp. 180-188 (2018).

15. Du, J., Zhu, J., Cook, W.D., and Huo, J. "DEA models for parallel systems: Game-theoretic approaches", Asia-Pacific Journal of Operational Research, 32(2), 1550008 (2015).

16. Fard, A.M.F. and Hajaghaei-Keshteli, M. "A tri-level location-allocation model for forward/reverse supply chain", Applied Soft Computing, 62, pp. 328-346 (2018).

17. Fard, A.M.F. and Hajiaghaei-Keshteli, M. "A biobjective partial interdiction problem considering different defensive systems with capacity expansion of facilities under imminent attacks", Applied Soft Computing, 68, pp. 343-359 (2018).

18. Fathollahi-Fard, A.M., Hajiaghaei-Keshteli, M., and Mirjalili, S. "Hybrid optimizers to solve a tri-level programming model for a tire closed-loop supply chain network design problem", Applied Soft Computing, 70, pp. 701-722 (2018).

19. Fathollahi-Fard, A.M., Hajiaghaei-Keshteli, M., and Tavakkoli-Moghaddam, R. "A bi-objective green home health care routing problem", Journal of Cleaner Production, 200, pp. 423-443 (2018).

20. Hajiaghaei-Keshteli, M. and Fathollahi-Fard, A.M. "A set of efficient heuristics and metaheuristics to solve a two-stage stochastic bi-level decision-making model for the distribution network problem", Computers \& Industrial Engineering, 123, pp. 378-395 (2018).

21. Färe, R., Grosskopf, S., Lovell, C.K., and Pasurka, C. "Multilateral productivity comparisons when some outputs are undesirable: a nonparametric approach", The Review of Economics and Statistics, 90(98) (1989).

22. Lu, W.M. and Lo, S.F. "A closer look at the economicenvironmental disparities for regional development in China", European Journal of Operational Research, 183(2), pp. 882-894 (2007).

23. Wang, K., Yu, S., and Zhang, W. "China's regional energy and environmental efficiency: A DEA window analysis based dynamic evaluation", Mathematical and Computer Modelling, 58(5-6), pp. 1117-1127 (2013).

24. Wu, J., Lv, L., Sun, J., and Ji, X. "A comprehensive analysis of China's regional energy saving and emission reduction efficiency: from production and treatment perspectives", Energy Policy, 84, pp. 166-176 (2015).

25. Sengupta, J.K. Dynamic of Data Envelopment Analysis: Theory of Systems Efficiency, Springer Science \& Business Media, Netherlands (1995).

26. Jafarian Moghaddam, A.R. and Ghoseiri, K. "Fuzzy dynamic multi-objective Data Envelopment Analysis model", Expert Systems with Applications, 38, pp. 850-855 (2011). 
27. Kawaguchi, H., Tone, K., and Tsutsui, M. "Estimation of the efficiency of Japanese hospitals using a dynamic and network data envelopment analysis model", Health Care Management Science, 17, pp. 101-112 (2014).

28. Wang, W., Lu, W., and Liu, P. "A fuzzy multiobjective two-stage DEA model for evaluating the performance of US bank holding companies", Expert Systems with Applications, 41, pp. 4290-4297 (2014).

29. Azizi, H. and Wang, Y.M. "Improved DEA models for measuring interval efficiencies of decision-making units", Measurement, 46(3), pp. 1325-1332 (2013).

30. Yang, X. and Morita, H. "Efficiency improvement from multiple perspectives: An application to Japanese banking industry", Omega, 41(3), pp. 501-509 (2013).

31. Amirteimoori, A. "DEA efficiency analysis: Efficient and anti-efficient frontier", Applied Mathematics and Computation, 186(1), pp. 10-16 (2007).

32. Jahed, R., Amirteimoori, A., and Azizi, H. "Performance measurement of decision-making units under uncertainty conditions: An approach based on double frontier analysis", Measurement, 69, pp. 264-279 (2015).

33. Doyle, J.R., Green, R.H., and Cook, W.D. "Upper and lower bound evaluation of multiattribute objects: Comparison models using linear programming", Organizational Behavior and Human Decision Processes, 64(3), pp. 261-273 (1995).

34. Badiezadeh, T., Saen, R.F., and Samavati, T. "Assessing sustainability of supply chains by double frontier network DEA: A big data approach", Computers \& Operations Research, 98, pp. 284-290 (2018).

35. Azizi, H., Kordrostami, S., and Amirteimoori, A. "Slacks-based measures of efficiency in imprecise data envelopment analysis: An approach based on data envelopment analysis with double frontiers", Computers \& Industrial Engineering, 79, pp. 42-51 (2015).

36. Wang, Y.M. and Lan, Y.X. "Estimating most productive scale size with double frontiers data envelopment analysis", Economic Modelling, 33, pp. 182-186 (2013).

37. Ransikarbum, K. and Mason, S.J. "Goal programmingbased post-disaster decision making for integrated relief distribution and early-stage network restoration", International Journal of Production Economics, 182, pp. 324-341 (2016).

38. Roy, S.K. and Maiti, S.K. "Stochastic bi level programming with multi-choice for Stackelberg game via fuzzy programming", International Journal of Operational Research, 29(4), pp. 508-530 (2017).

39. Roy, S.K. and Maity, G. "Minimizing cost and time through single objective function in multi-choice interval valued transportation problem", Journal of Intelligent \& Fuzzy Systems, 32(3), pp. 1697-1709 (2017).

40. Shabanpour, H., Yousefi, S., and Farzipoor Saen, R. "Future planning for benchmarking and ranking sustainable suppliers using goal programming and robust double frontiers DEA", Transportation Research
Part D: Transport and Environment, 50, pp. 129-143 (2017).

41. Dhahri, I. and Chabchoub, H. "Nonlinear goal programming models quantifying the bullwhip effect in supply chain based on ARIMA parameters", Eur $J$ Oper Res, 117 (3) pp. 1800-1810 (2007).

42. Yousefi, S., Soltani, R., Saen, R.F., and Pishvaee, M.S. "A robust fuzzy possibilistic programming for a new network GP-DEA model to evaluate sustainable supply chains", Journal of Cleaner Production, 166, pp. $537-549$ (2017).

43. Maiti, S.K. and Roy, S.K. "Bi-level programming for Stackelberg game with intuitionistic fuzzy number: A ranking approach", Journal of the Operations Research Society of China, 25(3), pp. 1-19 (2019).

44. Maiti, S.K. and Roy, S.K. "Multi-choice stochastic bilevel programming problem in cooperative nature via fuzzy programming approach", Journal of Industrial Engineering International, 12(3), pp. 287-298 (2016).

45. Chen, L.H., Ko, W.C., and Yeh, F.T. "Approach based on fuzzy goal programing and quality function deployment for new product planning", European Journal of Operational Research, 259(2), pp. 654-663 (2017).

46. Trivedi, A. and Singh, A. "A hybrid multi-objective decision model for emergency shelter location-relocation projects using fuzzy analytic hierarchy process and goal programming approach", International Journal of Project Management, 35(5), pp. 827-840 (2017).

47. He, Y., Gao, S., Liao, N., and Liu, H. "A nonlinear goal-programming-based DE and ANN approach to grade optimization in iron mining", Neural Computing and Applications, 27(7), pp. 2065-2081 (2016).

48. Roy, S.K., Maity, G., and Weber, G.W. "Multiobjective two-stage grey transportation problem using utility function with goals", Central European Journal of Operations Research, 25(2), pp. 417-439 (2017).

49. Roy, S.K., Maity, G., Weber, G.W., and Gök, S.Z.A. "Conic scalarization approach to solve multi-choice multi-objective transportation problem with interval goal", Annals of Operations Research, 253(1), pp. 599620 (2017).

50. Jolai, F., Yazdian, S.A., Shahanaghi, K., and Khojasteh, M.A. "Integrating fuzzy TOPSIS and multiperiod goal programming for purchasing multiple products from multiple suppliers", Journal of Purchasing and Supply Management, 17(1), pp. 42-53 (2011).

51. Wang, Y.M., Greatbanks, R., and Yang, J.B. "Interval efficiency assessment using data envelopment analysis", Fuzzy Sets and Systems, 153(3), pp. 347-370 (2005).

52. Wang, Y.M. and Chin, K.S. "A new approach for the selection of advanced manufacturing technologies: DEA with double frontiers", International Journal of Production Research, 47(23), pp. 6663-6679 (2009).

53. Kao, C. and Hwang, S.N. "Efficiency decomposition in two-stage data envelopment analysis: An application 
to non-life insurance companies in Taiwan", European Journal of Operational Research, 185(1), pp. 418-429 (2008).

54. Fu, Y., Tian, G., Fathollahi-Fard, A.M., Ahmadi, A., and Zhang, C. "Stochastic multi-objective modelling and optimization of an energy-conscious distributed permutation flow shop scheduling problem with the total tardiness constraint", Journal of Cleaner Production, 226, pp. 515-525 (2019).

55. Fathollahi-Fard, A.M., Hajiaghaei-Keshteli, M., and Mirjalili, S. "A set of efficient heuristics for a home healthcare problem", Neural Computing and Applications, 32(10), pp. 6185-6205 (2020).

\section{Biographies}

Ehsan Vaezi is a PhD student of Industrial Engineering at the College of Engineering, Science and Research Branch, Islamic Azad University, Tehran, Iran. He obtained his BS degree in Electrical Engineering from Iran University of Science and Technology (2009) and his MS degree in Industrial Engineering from Tehran University (2011). His research interests include: Game theory, data envelopment analysis, multi-criteria decision making techniques and system dynamics.

Seyyed Esmaeil Najafi received his BS degree in Mechanical Engineering from Shahid Beheshti University (College of Shahid Abbaspour) in Tehran (1998) and his MS degree in Industrial Engineering from Arak Islamic Azad University (2005). He obtained his $\mathrm{PhD}$ in Industrial Engineering from the Science and Research Branch of the Islamic Azad University (2010), where he is currently Assistant Professor in the School of Industrial Engineering.. He is Chief Editor of two international journals. His research interests include: multi-criteria decision making techniques, DEA, math- ematics modeling, and CRM. He has published several papers in refereed journals and conferences.

Seyyed Mohammad Hajimolana is Assistant Professor in the Department of Industrial Engineering at the University of Science and Research. He received his $\mathrm{PhD}$ in Industrial Engineering from Amirkabir University of Technology, Iran. His research interests are supply chain network design, inventory management, and applied operations research under uncertainty.

Farhad Hosseinzadeh Lotfi received his BS degree in Mathematics from Yazd University in 1991, and his MS degree from the Applied Mathematics Department at the University of Lahijan in 1995. He was awarded a PhD in Applied Mathematics from the Islamic Azad University, Science and Research Branch, Tehran by Professor Gholamreza Jahanshahlo in 1999. He has been Professor in the Department of Mathematics at the Islamic Azad University, Research and Science Branch, Tehran, since 2009. His research projects are in the following fields of specialization: Complementary problems in OR, data envelopment analysis and model building data envelopment analysis and its application, multiple criteria decision making and fuzzy sets and systems. He has published 11 books and more than 180 journal papers.

Mahnaz Ahadzadeh Namin received her $\mathrm{PhD}$ in Applied Mathematics from the Science and Research Branch of the Islamic Azad University, Tehran, Iran, in 2009. She is currently Assistant Professor in the Islamic Azad University, Shahr-e-Qods Branch. Her research interests are data envelopment analysis, efficiency analysis, applied mathematics, productivity analysis, and applied econometrics. Some of her publications are available from. 